Article

Doi 10.5943/mycosphere/6/6/5
ISSN 20777019

Mycosphere Online Edition

\title{
New asexual morph taxa in Phaeosphaeriaceae
}

\section{Li WJ ${ }^{1,2,3,4}$, Bhat $\mathrm{DJ}^{5}$, Camporesi $\mathrm{E}^{6}$, Tian $\mathrm{Q}^{3,4}$, Wijayawardene $\mathrm{NN}^{3,4}$, Dai DQ $\mathbf{D Q}^{3,4}$, Phookamsak $R^{3,4}$, Chomnunti $P^{3,4}$ Bahkali AH $^{7}$ \& Hyde KD $^{1,2,3,7^{*}}$}

\author{
${ }^{1}$ World Agroforestry Centre, East and Central Asia, 132 Lanhei Road, Kunming 650201, China \\ ${ }^{2}$ Key Laboratory of Economic Plants and Biotechnology, Kunming Institute of Botany, Chinese Academy of Sciences, \\ Lanhei Road No 132, Panlong District, Kunming, Yunnan Province, 650201, PR China \\ ${ }^{3}$ Center of Excellence in Fungal Research, Mae Fah Luang University, Chiang Rai 57100, Thailand \\ ${ }^{4}$ School of Science, Mae Fah Luang University, Chiang Rai 57100, Thailand \\ ${ }^{5}$ Formerly, Department of Botany, Goa University, Goa 403206, India \\ ${ }^{6}$ A.M.B. GruppoMicologicoForlivese "Antonio Cicognani", Via Roma 18, Forli, Italy \\ ${ }^{7}$ Botany and Microbiology Department, College of Science, King Saud University, Riyadh, KSA 11442, Saudi Arabia
}

Li WJ, Bhat DJ, Camporesi E, Tian Q, Wijayawardene NN, Dai DQ³, Phookamsak R, Chomnunti P, Bahkali AH, Hyde KD 2015 - New asexual morph taxa in Phaeosphaeriaceae. Mycosphere 6(6), 681-708, Doi 10.5943/mycosphere/6/6/5

\begin{abstract}
Species of Phaeosphaeriaceae, especially the asexual taxa, are common plant pathogens that infect many important economic crops. Ten new asexual taxa (Phaeosphaeriaceae) were collected from terrestrial habitats in Italy and are introduced in this paper. In order to establish the phylogenetic placement of these taxa within Phaeosphaeriaceae we analyzed combined ITS and LSU sequence data from the new taxa, together with those from GenBank. Based on morphology and molecular data, Poaceicola gen. nov. is introduced to accommodate the new species Po. arundinis (type species), Po. bromi and Po. elongata. The new species Parastagonospora dactylidis, P. minima, P. italica, $P$. uniseptata and P. allouniseptata, Septoriella allojunci and Wojnowicia spartii are also introduced with illustrated accounts and compared with similar taxa. We also describe an asexual morph of a Nodulosphaeria species for the first time.
\end{abstract}

Key words - Asexual morphs - Phaeosphaeriaceae - Phylogeny - Taxonomy

\section{Introduction}

The order Pleosporales has been of great research interest in the recent years and has undergone considerable revision (Zhang et al. 2009, 2012, Hyde et al. 2013, Ariyawansa et al. 2014, 2015b). Hyde et al. (2013) provided a taxonomic account for 41 families in Pleosporales, in which Phaeosphaeriaceae is a large and important family, comprising more than 300 species, and has also undergone considerable revision (Hyde et al. 2013, Phookamsak et al. 2014, Liu et al. 2015). Species in Phaeosphaeriaceae can be saprobic or endophytic, but many are pathogenic on economically 
important plants and crops (Kirk et al. 2008, Zhang et al. 2009, Quaedvlieg et al. 2013, Hyde et al. 2013, Wijayawardene et al. 2014, Phookamsak et al. 2014). For example, Parastagonospora species are pathogenic on Poaceae and are directly or indirectly responsible for significant crop losses in wheat, barley and rye worldwide (Quaedvlieg et al. 2013). In addition, many genera, such as, Phaeosphaeriopsis, Setophoma, Xenoseptoria and Wojnowicia have been found associated with leaf spot disease of various hosts (Arzanlou and Crous 2006, Quaedvlieg et al. 2013; Wijayawardene et al. 2013; Phookamsak et al. 2014).

Barr (1979a) introduced Phaeosphaeriaceae based on the type genus Phaeosphaeria, and the history has been reviewed by Phookamsak et al. (2014). The latter authors also carried out a revision of Phaeosphaeriaceae, based on multi-gene (ITS, LSU, SSU, RPB2 and TEF1) analyses coupled with morphological data. They included 30 genera in the family, in which 17 genera are asexual morphs, Liu et al. (2015) and Ariyawansa et al. (2015c) added four additional genera in Phaeosphaeriaceae, and to date, 37 genera are accommodated in the family (Table 1).

In the present study we introduce ten new asexual taxa in Phaeosphaeriaceae using combined ITS and LSU sequence data, as well as morphological data.

\section{Material \& methods}

\section{Collection and examination of specimens}

Fresh specimens were collected from terrestrial habitats in Italy. Samples were examined and pure cultures obtained by single spore isolation following the method described in Chomnunti et al. (2014). The growing colonies were transferred to $2 \%$ potato-dextrose agar (PDA) and incubated at $25^{\circ} \mathrm{C}$. The colony characters and growth rates were determined after one to four weeks. The pure cultures from our study are deposited at Mae Fah Luang University Culture Collection (MFLUCC). Duplicate cultures are deposited in the International Collection of Microorganisms from Plants, Landcare Research, New Zealand (ICMP) and Kunming Institute of Botany Culture Collection (KUMCC). The holotype is deposited at the herbarium of Mae Fah Luang University (MFLU), Chiang Rai, Thailand, and the isotype specimens are deposited at the herbarium of Kunming institute of Botany Chinese Academy of Sciences (KUN). Faces of Fungi numbers and Index Fungorum numbers were obtained as explained in Jayasiri et al. (2015). The asexual morphs were established in culture using the method of Phookamsak et al. (2015).

\section{DNA extraction, PCR amplification and sequencing}

Isolates were grown on PDA plates in the darkness at $25^{\circ} \mathrm{C}$ until completely covering the agar surface. The mycelium (about $50 \mathrm{mg}$ ) was scraped off and collected in a $1.5 \mathrm{ml}$ micro centrifuge tube. Genomic DNA was extracted from fresh mycelium, following the specification of Biospin Fungus Genomic DNA Extraction Kit (BioFlux®). For Poaceicola arundinis sp. nov. and Septoriella allojunci sp. nov., despite several attempts we could not isolate them into culture. Therefore, DNA was extracted directly from the conidiomata of the dried specimens following the method of Li et al. (2015). The primer pairs LROR and LR5 as defined by Vilgalys and Hester (1990) were used to amplify a segment of the large subunit rDNA. Primer pairs ITS4 and ITS5 as defined by White et al. (1990) were used to amplify the internal transcribed spacers (ITS). DNA amplification was performed by polymerase chain reaction (PCR). The sequencing of PCR products were carried at Shang Hai Biological Engineering Technology Co. Ltd (Shang Hai, P. R. China).

\section{DNA sequence data analysis}

Sequences were obtained from GenBank mostly following previous literature (Phookamsak et al. 2014, Ariyawansa et al. 2014, 2015c, Crous et al. 2015b, Liu et al. 2015) and are listed in Table 2. 
Multiple sequences were aligned using Bioedit v. 7.0.9 (Hall, 1999) and Clustal X v. 1.83 (Thompson et al., 1997). The alignments were checked visually and improved manually wherever necessary. A maximum likelihood (ML) analysis was performed with raxmlGUI version 1.3 (Silvestro \& Michalak 2011). The optimal ML tree search was conducted with 1000 separate runs, using the default algorithm of the program from a random starting tree for each run. The final tree was selected among suboptimal trees from each run by comparing likelihood scores under the GTRGAMMA substitution model. The resulting trees were printed with TreeView v. 1.6.6 (Page, 1996).

\section{Results}

\section{Phylogenetic analyses}

ITS and LSU sequence data were used to resolve the generic placement of the collections of Phaeosphaeriaceae. The alignment datasets included 83 taxa of which Didymella exigua was used as the outgroup taxon. The combined datasets comprised 1435 characters including gaps. The best scoring RAxML tree was chosen as the backbone tree and is shown in Figure 1.

The phylogenetic analyses show that Poaceicola elongata comb. nov. and two other species, Po. arundinis and Po. bromi, are represented as a lineage distinct from any other genera in Phaeosphaeriaceae (Fig. 1). The other strains are distributed in Nodulosphaeria (one species), Parastagonospora (five species), and Septoriella (one species). Based on the morphology and sequence data, these fresh collections are introduced as new asexual taxa in Phaeosphaeriaceae. The new taxa are five species of Parastagonospora, i.e. $P$. allouniseptata, $P$. dactylidis, $P$. minima, $P$. talica and P. uniseptata; Septoriella allojunci sp. nov, and an asexual morph of Nodulosphaeria, $N$. digitalis.

\section{Taxonomy}

Nodulosphaeria digitalis W.J. Li, Camporesi, D.J. Bhat \& K.D. Hyde, sp. nov.

Fig. 2 Index Fungorum number: IF551664

Facesoffungi number: FoF 01302,

Etymology - Named after the host genus, Digitalis.

Holotype - MFLU 15-2716

Saprobic on dead stems of Digitalis sp., forming conspicuous, rounded, black fruiting bodies. Sexual morph - Undetermined. Asexual morph - Coelomycetous. Conidiomata 130-150 $\mu \mathrm{m}$ high, 240-270 $\mu \mathrm{m}$ diam., pycnidioid, brown, solitary, aggregated or confluent, semi-immersed, subglobose, unilocular, papillate. Ostiole centrally located, short. Wall of conidiomata 20-60 $\mu \mathrm{m}$ wide, composed thick-walled, brown outer cells of textura angularis, and an inner layers of hyaline textura prismatica. Conidiophores cylindrical, short, hyaline, arrising from the inner layer of conidioma. Conidiogenous cells 7-15 × 2-4 $\mu \mathrm{m}$, entoblastic, phialidic, cylindrical to subcylindrical, hyaline, smooth-walled. Conidia 9-18 $\times 2-9 \mu \mathrm{m}(\bar{x}=15 \times 3 \mu \mathrm{m} ; \mathrm{n}=30)$, hyaline, falcate, fusiform, strongly curved towards the apices, rounded at both ends, 0-1-septate, smooth and thick-walled.

Material examined - ITALY, Province of Arezzo [AR], Bagno di Cetica, on dead stem of Dactylis sp., 1 October 2012, Erio Camporesi, IT-753 (MFLU 15-2716); ibid. (KUN! HKAS 90731).

Notes - Nodulosphaeria digitalis clustered with Nodulosphaeria species with high bootstrap suppert (99\%), but $N$. digitalis formed a separate branch basal to N. modesta (Rabenh.) Munk ex L. Holm) and N. italica (Fig. 1). This is the first recorded asexual morph for Nodulosphaeria. 
Table 1 Genera of Phaeosphaeriaceae, types species and morphs

\begin{tabular}{|c|c|c|c|}
\hline Genus name & Types species & Sexual/Asexual morph & Reference \\
\hline Allophaeosphaeria & Allophaeosphaeria muriformia & Asexual morph: Coelomytous & Liu et al. 2015 \\
\hline Amarenographium & Amarenographium metableticum & Sexual morph unknown & Phookamsak et al. 2014 \\
\hline Amarenomyces & Amarenomyces ammophilae & Asexual morph unknown & Phookamsak et al. 2014 \\
\hline Ampelomyces & Ampelomyces quisqualis & Sexual morph unknown & Phookamsak et al. 2014 \\
\hline Bricookea & Bricookea sepalorum & Asexual morph unknown & Phookamsak et al. 2014 \\
\hline Chaetosphaeronema & Chaetosphaeronema hispidulum & Sexual morph unknown & De Gruyter et al. 2010 \\
\hline Dematiopleospora & Dematiopleospora mariae & Asexual morph unknown & Wanasinghe et al. 2014 \\
\hline Diederichomyces & Diederichomyces xanthomendozae & Sexual morph unknown & Trakunyingcharoe et al. 2014 \\
\hline Dothideopsella & Dothideopsella agminalis & Asexual morph unknown & Wijayawardene et al. 2014b \\
\hline Entodesmium & Entodesmium rude & Asexual morph unknown & Zhang et al. 2009 \\
\hline Eudarluca & Eudarluca australis & Asexual morph unknown & Wijayawardene et al. 2014b \\
\hline Galliicola & Galliicola pseudophaeosphaeria & Asexual morph unknown & Ariyawansa et al. $2015 \mathrm{~b}$ \\
\hline Leptospora & Leptospora porphyrogona & Asexual morph unknown & Crous et al. 2006 \\
\hline Loratospora & Loratospora aestuari & Asexual morph unknown & Schoch et al. 2009 \\
\hline Neosetophoma & Neosetophoma samararum & Sexual morph unknown & De Gruyter et al. 2010 \\
\hline Neostagonospora & Neostagonospora caricis & Sexual morph unknown & Quaedvlieg et al.2013 \\
\hline Nodulosphaeria & Nodulosphaeria hirta & Asexual morph: Coelomytous & from this study \\
\hline Ophiobolus & Ophiobolus disseminans & Asexual morph unknown & Phookamsak et al. 2014 \\
\hline Ophiosphaerella & Ophiosphaerella graminicola & Asexual morph unknown & Zhang et al. 2012 \\
\hline Paraphoma & Paraphoma radicina & Sexual morph unknown & Gruyter et al. 2010 \\
\hline Parastagonospora & Parastagonospora nodorum & Sexual morph $=$ phaeosphaeria-like & Quaedvlieg et al. 2013 \\
\hline Phaeosphaeria & Phaeosphaeria oryzae & Asexual morph $=$ Phaeoseptoria & Quaedvlieg et al. 2013 \\
\hline Phaeosphaeriopsis & Phaeosphaeriopsis glaucopunctata & Asexual morph Coelomycetous & Phookamsak et al. 2014 \\
\hline Phaeostagonospora & Phaeostagonospora nolinae & Sexual morph unknown & Phookamsak et al. 2014 \\
\hline Poaceicola & Poaceicola arundinis & Sexual morph= Phaeosphaeria-like & from this study \\
\hline Populocrescentia & Populocrescentia forlicesenensis & Sexual morph unknown & Ariyawansa et al. 2015 b \\
\hline Sclerostagonospora & Sclerostagonospora heraclei & Sexual morph $=$ phaeosphaeria-like & Quaedvlieg et al. 2013 \\
\hline Scolecosporiella & Scolecosporiella typhae & Asexual morph unknown & Phookamsak et al. 2014 \\
\hline Scolicosporium & Scolicosporium fagi & Sexual morph unknown & Wijayawardene et al. 2013 \\
\hline Septoriella & Septoriella phragmitis & Sexual morph unknown & Crous et al. 2015 \\
\hline
\end{tabular}




\begin{tabular}{llll}
\hline Genus name & Types species & Sexual/Asexual morph & Reference \\
\hline Setomelanomma & Setomelanomma holmii & Sexual morph unknown & Phookamsak et al. 2014 \\
Setophoma & Setophoma terrestris & $\begin{array}{l}\text { The sexual morph of Setophoma } \\
\text { is similar to Phaeosphaeria species }\end{array}$ & Phookamsak et al. 2014 \\
Stagonospora & - & Sexual morph unknown & Phookamsak et al. 2014 \\
Sulcispora & Sulcispora pleurospora & Asexual morph unknown & Senanayake et al. 2014 \\
Tiarospora & Tiarospora westendorpii & Sexual morph unknown & Phookamsak et al. 2014 \\
Vrystaatia & Vrystaatia aloeicola & Sexual morph unknown & Quaedvlieg et al. 201 \\
Wojnowicia & Wojnowicia hirta & Sexual morph unknown & Crous et al. 2015 \\
Wojnowiciella & Wojnowiciella eucalypti & Sexual morph unknown & Crous et al. 2015a \\
Xenophoma & Xenophoma puncteliae & Sexual morph unknown & Phookamsak et al. 2014 \\
Xenoseptoria & Xenoseptoria neosaccardoi & Sexual morph unknown & Quaedvlieg et al. 2013 \\
\hline
\end{tabular}

Table 2 Collection details and GenBank accession number of isolates includes in this study. The newly generated sequences are indicated in bold, T signifies ex-type/ex-epitype isolates.

\begin{tabular}{|c|c|c|c|c|c|}
\hline \multirow[t]{2}{*}{ Species name } & \multirow[t]{2}{*}{ strain } & \multirow[t]{2}{*}{ Host } & \multirow[t]{2}{*}{ Country } & \multicolumn{2}{|l|}{$\begin{array}{l}\text { GenBank } \\
\text { accession } \\
\text { number }\end{array}$} \\
\hline & & & & ITS & LSU \\
\hline Allophaeosphaeria muriformia & MFLUCC $13-0349^{\mathrm{T}}$ & - & Italy & KP765680 & KP765681 \\
\hline Allophaerosphaeria subcylindrospora & MFLUCC $13-0380^{\mathrm{T}}$ & Dactylis glomerata & Italy & KT314184 & KT314183 \\
\hline Ampelomyces quisqualis & CBS 129.79 & - & Canada & HQ108038 & JX681064 \\
\hline Chaetosphaeronema coonsii & CBS 559.78 & Malus sylvestris & Japan & - & EU754196 \\
\hline \multirow{2}{*}{$\begin{array}{l}\text { Dematiopleospora mariae } \\
\text { Didymella exigua }\end{array}$} & MFLUCC $13-0612^{\mathrm{T}}$ & Ononis spinosa & Italy & - & KJ749653 \\
\hline & CBS $183.55^{\mathrm{T}}$ & Rumex arifolius & France & GU237794 & EU754155 \\
\hline Diederichomyces caloplacae & CBS 129338 & Caloplaca cerina & Canada & KP170639 & JQ238643 \\
\hline Diederichomyces cladoniicola & CBS 128026 & Cladonia sp. & Spain & KP170642 & JQ238628 \\
\hline Diederichomyces ficuzzae & CBS 128019 & Ramalina fastigiata & France & KP170647 & JQ238616 \\
\hline Entodesmium rude & CBS 650.86 & Unknown & - & - & GU301812 \\
\hline Galliicola pseudophaeosphaeria & MFLUCC $14-0527^{\mathrm{T}}$ & Galium sp. & Italy & KT326692 & KT326693 \\
\hline Leptospora rubella & CPC 11006 & Eucalyptus sp. & Colombia & DQ195780 & DQ195792 \\
\hline Loratospora aestuarii & JK 5535B & Juncus roemerianus & USA & - & GU301838 \\
\hline Neosetophoma samarorum & CBS $138.96^{\mathrm{T}}$ & Phlox paniculata & Netherlands & FJ427061 & KF251664 \\
\hline Neostagonospora caricis & CBS 135092/S616 ${ }^{\mathrm{T}}$ & Carex acutiformis & Netherlands & KF251163 & KF251667 \\
\hline Neostagonospora elegiae & CBS $135101^{\mathrm{T}}$ & Elegia cuspidata & South Africa & KF251164 & KF251668 \\
\hline
\end{tabular}




\begin{tabular}{|c|c|c|c|c|c|}
\hline \multirow[t]{2}{*}{ Species name } & \multirow[t]{2}{*}{ strain } & \multirow[t]{2}{*}{ Host } & \multirow[t]{2}{*}{ Country } & \multicolumn{2}{|l|}{$\begin{array}{l}\text { GenBank } \\
\text { accession } \\
\text { number }\end{array}$} \\
\hline & & & & ITS & LSU \\
\hline Nodulosphaeria aconiti & MFLUCC 13-0728 & Aconitum vulparia & Italy & & \\
\hline Nodulosphaeria digitalis & - & Dactylis sp. & Italy & KU058710 & KU058720 \\
\hline Nodulosphaeria dolioloides & MFLUCC 15-0065 & Achillea sp. & Italy & - & - \\
\hline Nodulosphaeria hirta & MFLUCC 13-0867 & Achillea sp.- & Italy & & \\
\hline Nodulosphaeria sanguisorbae & MFLUCC 13-0745 & Sanguisorba minor & Italy & & \\
\hline Nodulosphaeria scabiosae & MFLUCC 14-1111 & Scabiosa sp. & Italy & & \\
\hline Nodulosphaeria senecionis & MFLUCC 15-1297 & Senecio sp. & Italy & KT290257 & KT290257 \\
\hline Nodulosphaeria spectabilis & MFLUCC 14-1112 & Peucedanum cervaria & Italy & - & - \\
\hline Ophiobolus cirsii & MFLUCC 13-0218 & - & Italy & KM014664 & KM014662 \\
\hline Ophiobolus disseminans & AS2L14-6 & - & - & KP117305 & - \\
\hline Ophiosphaerella agrostidis & MFLUCC 12-0007 & grass & Thailand & KM434272 & KM434282 \\
\hline Ophiosphaerella agrostidis & MFLUCC 11-0152 & grass & Thailand & KM434271 & KM434281 \\
\hline \multirow{2}{*}{$\begin{array}{l}\text { Paraphoma chrysanthemicola } \\
\text { Paraphoma radicina }\end{array}$} & \multirow{2}{*}{$\begin{array}{l}\text { CBS } 522.66 \\
\text { CBS } 111.79^{\mathrm{T}}\end{array}$} & Chrysanthemum morifolium & United Kingdom & FJ426985 & KF251670 \\
\hline & & Malus sylvestris & Netherlands & KF251172 & KF251676 \\
\hline Parastagonospora allouniseptata & MFLUCC 13-0386 ${ }^{\mathrm{T}}$ & Dactylis glomerata & Italy & KU058711 & KU058721 \\
\hline Parastagonospora avenae & CBS 289.69 & Lolium perenne & Germany & KF251174 & KF251678 \\
\hline Parastagonospora avenae & CBS 290.69 & Lolium perenne & Germany & KF251175 & KF251679 \\
\hline Parastagonospora caricis & CBS $135671 / \mathrm{S} 615^{\mathrm{T}}$ & Carex acutiformis & Netherlands & KF251176 & KF251680 \\
\hline Parastagonospora dactylidis & MFLUCC 13-0375 ${ }^{\mathrm{T}}$ & Dactylis sp. & Italy & KU058712 & KU058722 \\
\hline Parastagonospora italica & MFLUCC 13-0377 ${ }^{\mathrm{T}}$ & Dactylis sp. & Italy & KU058714 & KU058724 \\
\hline Parastagonospora minima & MFLUCC 13-0376 ${ }^{\mathrm{T}}$ & Dactylis sp. & Italy & KU058713 & KU058723 \\
\hline Parastagonospora nodorum & CBS 110109 & Lolium perenn & Denmark & KF251177 & KF251681 \\
\hline Parastagonospora poae & CBS 135091 & Poa sp. & Netherlands & KF251179 & KF251683 \\
\hline Parastagonospora poae & CBS $135089^{\mathrm{T}}$ & Poa sp. & Netherlands & KF251178 & KF251682 \\
\hline Parastagonospora poagena & CBS $136776^{\mathrm{T}}$ & Poa sp. & Netherlands & KJ869116 & KJ869174 \\
\hline Parastagonospora uniseptata & MFLUCC 13-038 $7^{\mathrm{T}}$ & Daucus sp. & Italy & KU058715 & KU058725 \\
\hline Phaeosphaeria papayae & CBS $135416 / \mathrm{S} 528^{\mathrm{T}}$ & Carica papaya & Brazil & KF251187 & KF251690 \\
\hline Phaeosphaeria alpina & CBS 456.84 & Phleum alpinum & Switzerland & KF251181 & KF251684 \\
\hline Phaeosphaeria chiangraina & MFLUCC $13-0231^{\mathrm{T}}$ & Oryza sativa & Thailand & KM434270 & KM434280 \\
\hline Phaeosphaeria eustoma & CBS 573.86 & Dactylis glomerata & Switzerland & AF439479 & - \\
\hline Phaeosphaeria eustoma & CBS 573.86 & Dactylis glomerata & Switzerland & AF439479 & - \\
\hline Phaeosphaeria musae & CBS $120026^{\mathrm{T}}$ & Unknown & Unknown & DQ885894 & GU301862 \\
\hline Phaeosphaeria nigrans & CBS 307.79 & Zea mays & Switzerland & KF251184 & KF251687 \\
\hline Phaeosphaeria oryzae & CBS $110110^{\mathrm{T}}$ & Oryza sativa & South Korea & KF251186 & KF251689 \\
\hline Phaeosphaeria thysanolaenicola & MFLUCC $10-0563^{\mathrm{T}}$ & Thysanolaena maxima Kuntze & Thailand & KM434266 & KM434276 \\
\hline
\end{tabular}




\begin{tabular}{|c|c|c|c|c|c|}
\hline \multirow[t]{2}{*}{ Species name } & \multirow[t]{2}{*}{ strain } & \multirow[t]{2}{*}{ Host } & \multirow[t]{2}{*}{ Country } & \multicolumn{2}{|l|}{$\begin{array}{l}\text { GenBank } \\
\text { accession } \\
\text { number }\end{array}$} \\
\hline & & & & ITS & LSU \\
\hline Phaeosphaeria typharum & CBS 296.54 & Nardus stricta & Switzerland & KF251192 & KF251695 \\
\hline Phaeosphaeriopsis glaucopunctata & MFLUCC $13-0265^{\mathrm{T}}$ & Ruscus aculeatus & Italy & KJ522473 & KJ522477 \\
\hline Phaeosphaeriopsis glaucopunctata & MFLUCC $13-0220$ & Ruscus hypoglossum & Italy & KJ522474 & KJ522478 \\
\hline Phaeosphaeriopsis triseptata & MFLUCC $13-0271^{\mathrm{T}}$ & Ruscus aculeatus & Italy & KJ522475 & KJ522479 \\
\hline Poaceicola arundinis & - & reed (Juncaceae) & Italy & KU058716 & KU058726 \\
\hline Poaceicola bromi & MFLUCC 13-0739 ${ }^{\mathrm{T}}$ & Bromus sterilis & Italy & KU058717 & KU058727 \\
\hline Poaceicola elongate & MFLUCC 12-4444 ${ }^{\mathrm{T}}$ & Unknown & Italy & KM491546 & KM491548 \\
\hline Populocrescentia forlicesenensis & MFLUCC $15-0651^{\mathrm{T}}$ & Populus nigra & Italy & KT306948 & KT306952 \\
\hline Sclerostagonospora cycadis & CBS $123538^{\mathrm{T}}$ & Cycas revolut & Japan & FJ372393 & FJ372410 \\
\hline Scolicosporium minkeviciusii & MFLUCC $12-0089^{\mathrm{T}}$ & Quercus pubescens & Italy & - & KF366382 \\
\hline Septoriella allojunci & - & Juncus sp. & Italy & KU058718 & KU058728 \\
\hline Septoriella hubertusii & CBS $338.86^{\mathrm{T}}$ & Phragmites australis & France & KF251230 & KF251733 \\
\hline Septoriella leuchtmannii & CBS 459.84 & Phragmites australis & Switzerland & KF251188 & KF251691 \\
\hline Septoriella oudemansii & CPC $24116^{\mathrm{T}}$ & Phragmites australis & Netherlands & - & KJ869224 \\
\hline Septoriella phragmitis & CPC $24118^{\mathrm{T}}$ & Phragmites sp. & Netherlands & KR873251 & KR873279 \\
\hline Septoriella poae & CBS $136766^{\mathrm{T}}$ & Poa sp. & Netherlands & KJ869111 & KJ869169 \\
\hline Setomelanomma holmii & CBS 110217 & Unknown & - & - & GU301871 \\
\hline Setophoma chromolaena & CBS 135105T/CPC 18553 & Chromolaena odorata & Brazil & KF251244 & KF251747 \\
\hline Setophoma sacchari & CBS 333.39 & Saccharum officinarum & Brazil & KF251245 & KF251748 \\
\hline Setophoma sacchari & MFLUCC 11-0154 & Saccharum officinarum & Thailand & KJ476144 & KJ476146 \\
\hline Stagonospora neglecta & CBS 343.86 & Phragmites australi & France & AJ496630 & EU754218 \\
\hline Sulcispora pleurospora & MFLUCC 13-0796 & Unknown & Italy & KP271443 & KP271444 \\
\hline Vagicola vagans & CBS $604.86^{\mathrm{T}}$ & Calamagrostis arundinacea & Sweden & KF251193 & KF251696 \\
\hline Vrystaatia aloeicola & CBS 135107/CPC $20617^{\mathrm{T}}$ & Aloe maculata & South Africa & KF251278 & KF251781 \\
\hline Wojnowicia dactylidicola & MFLUCC $13-0738^{\mathrm{T}}$ & Dactylis glomerata & Italy & KP744469 & KP684147 \\
\hline Wojnowicia lonicerae & MFLUCC $13-0737^{\mathrm{T}}$ & Lonicerasp & Italy & KP744471 & KP684151 \\
\hline Wojnowicia spartii & MFLUCC 13-0402 ${ }^{\mathrm{T}}$ & Spartium sp. & Italy & KU058719 & KU058729 \\
\hline Wojnowicia dactylidis & MFLUCC $13-0735^{\mathrm{T}}$ & Dactylis glomerata & Italy & KP744470 & KP684149 \\
\hline Wojnowiciella eucalypti & CPC $25024^{\mathrm{T}}$ & Eucalyptus grandis & Colombia & KR476741 & KR476774 \\
\hline Wojnowiciella viburni & MFLUCC $12-0733^{\mathrm{T}}$ & Viburnum utile & China & KC594286 & KC594287 \\
\hline Xenophoma puncteliae & CBS $128022^{\mathrm{T}}$ & Punctelia rudecta & USA & JQ238617 & JQ238619 \\
\hline Xenoseptoria neosaccardoi & CBS $128665^{\mathrm{T}}$ & Lysimachia vulgaris var. Davurica & South Korea & KF251281 & KF251784 \\
\hline Xenoseptoria neosaccardoi & CBS 120.43 & Cyclamen persicum & Netherlands & KF251280 & KF251783 \\
\hline
\end{tabular}




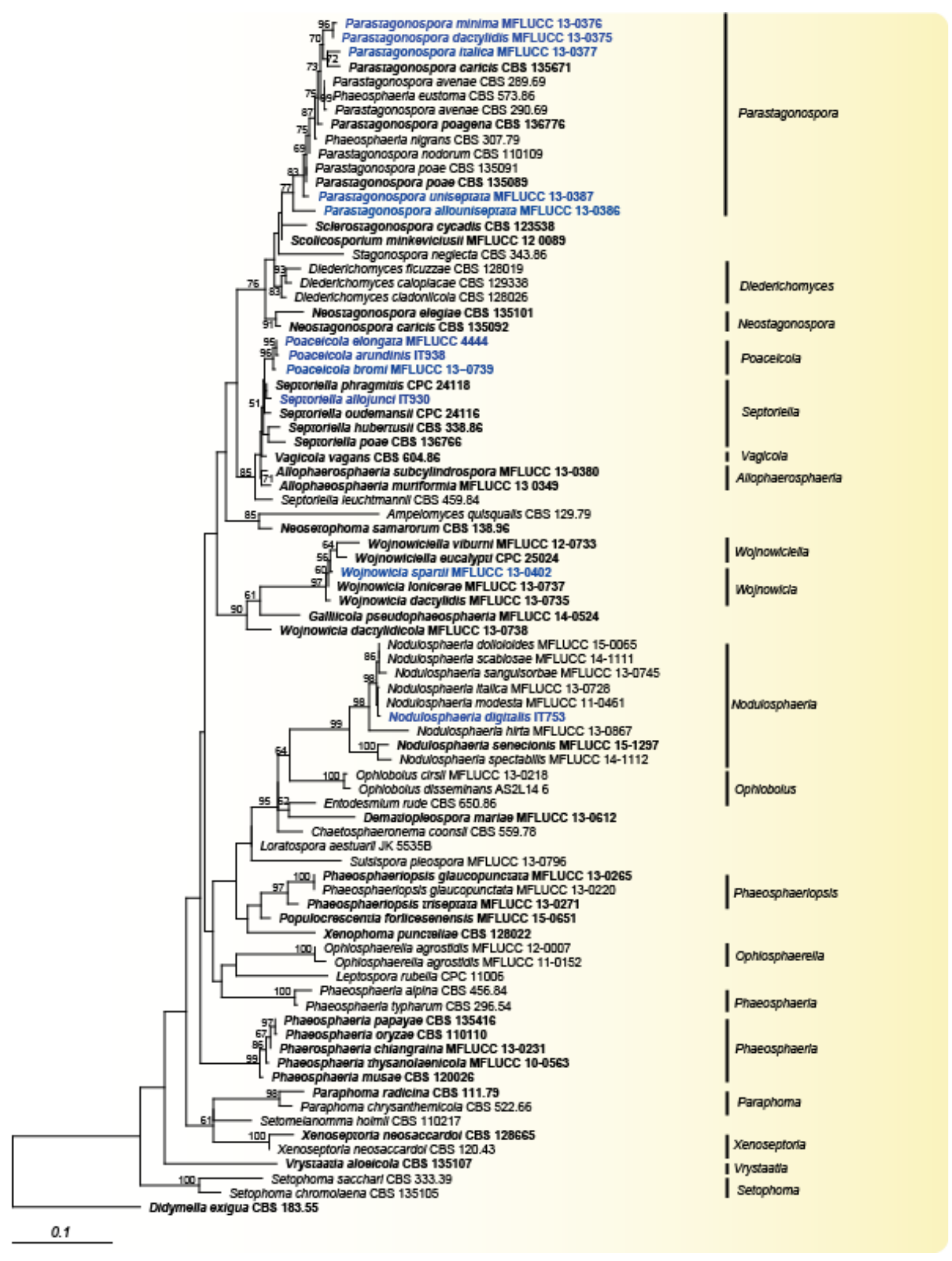

Fig. 1 - Best scoring RAxML tree of Phaeosphaeriaceae strains obtained from analysis of ITS and LSU sequence data. RAxML bootstrap support values (equal to or greater than $50 \%$ based on 1.000 replicates) are shown at the nodes. The ex-type strains are in bold; the new isolates are in blue. The tree is rooted to Didymella exigua CBS 183.55. 


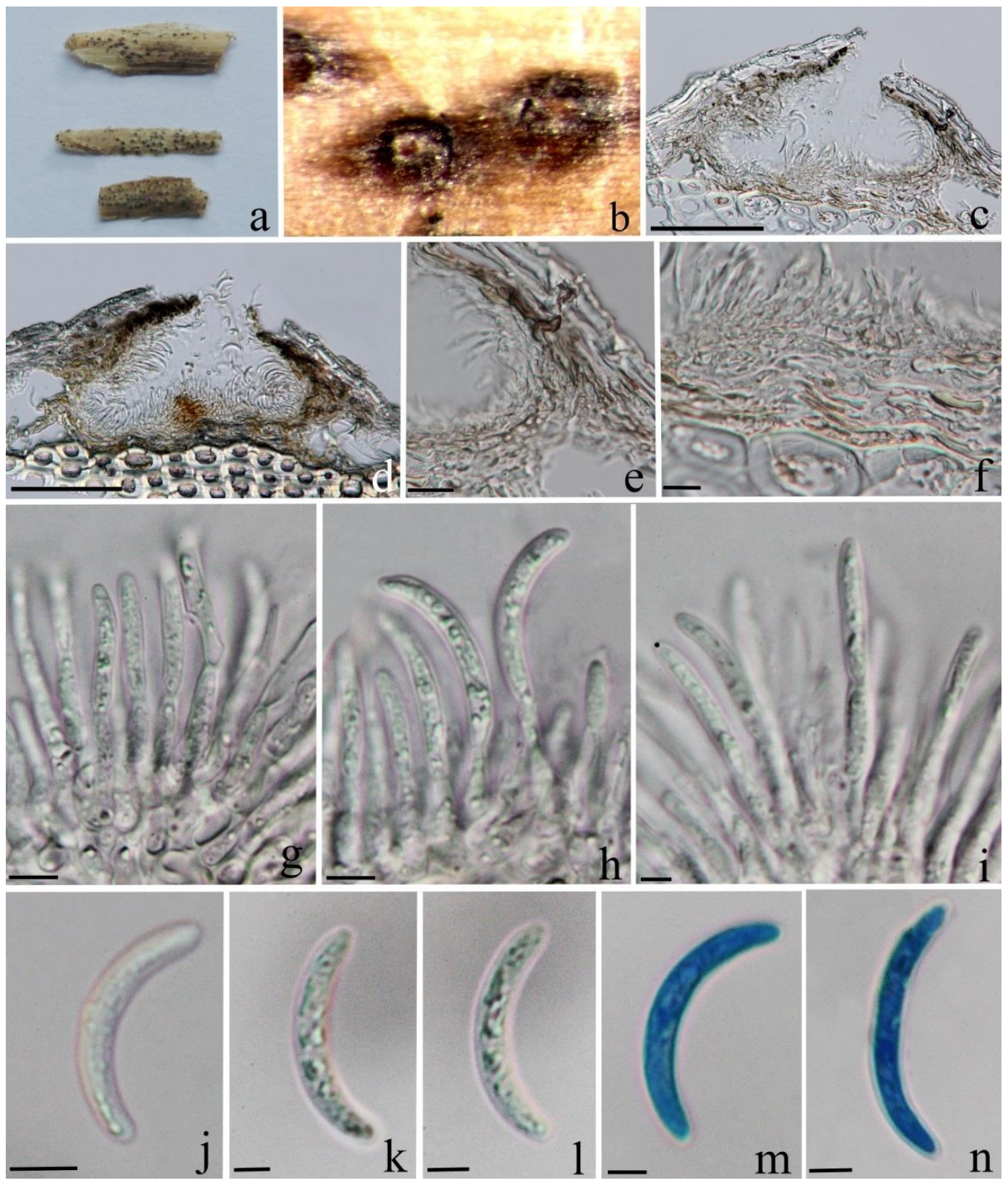

Fig. 2 - Nodulosphaeria digitalis (MFLU 15-2716, holotype) a Herbarium specimen b Appearance of black conidiomata on the host. c, d Vertical section of conidioma. e, f Section of peridium. $g-i$ Conidiogenous cells and developing conidia. $\mathrm{g}-\mathrm{n}$ Conidia. - Bars: $\mathrm{c}-\mathrm{d}=100 \mu \mathrm{m} ; \mathrm{e}-\mathrm{f}=20 \mu \mathrm{m} ; \mathrm{g}-\mathrm{i}=5$ $\mu \mathrm{m} ; \mathrm{j}-\mathrm{n}=5 \mu \mathrm{m}$.

Parastagonospora allouniseptata W.J. Li, Camporesi, D.J. Bhat \& K.D. Hyde, sp. nov. Fig. 3 Index Fungorum number: IF551665

Facesoffungi number: FoF 01307

Etymology - Named after its morphological similarity to Parastagonospora uniseptata

Holotype - MFLU 15-0698

Saprobic on dead stem of Dactylis glomerata L. (Poaceae), forming conspicuous, rounded, black fruiting bodies. Sexual morph - Undetermined. Asexual morph - Coelomycetous. Conidiomata 60-90 $\mu \mathrm{m}$ high, 70-90 $\mu \mathrm{m}$ diam., pycnidial, black, solitary or gregarious, semi- 

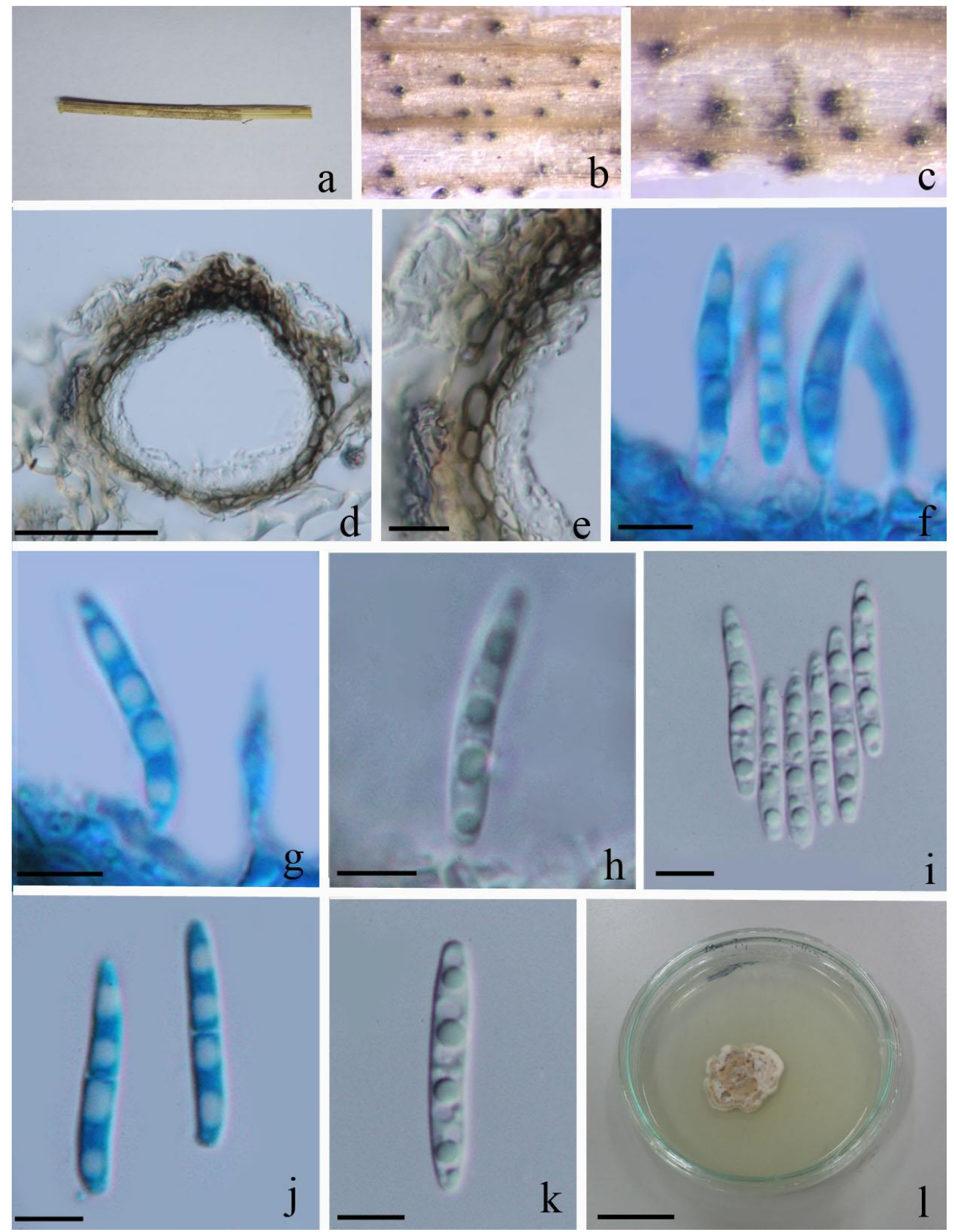

Fig. 3 - Parastagonospora allouniseptata (MFLU 15-0698, holotype) a Herbarium specimen. b-c Appearance of black conidiomata on the host. d Vertical section of conidioma. e Section of peridium. $\mathrm{f}-\mathrm{h}$ Conidiogenous cells and developing conidia. i-k Conidia. 1 Culture on PDA. - Bars: $\mathrm{d}=50 \mu \mathrm{m}$; e $=10 \mu \mathrm{m} ; \mathrm{f}-\mathrm{k}=5 \mu \mathrm{m} ; \mathrm{l}=25 \mathrm{~mm}$.

immersed, unilocular, globose to subglobose, ostiolate. Ostiole centrally located, papillate. Wall of conidiomata 5-10 $\mu \mathrm{m}$ wide, comprising outer layer of brown to dark brown, thin-walled cell of textura globosa, and an inner layer of light brown to hyaline cell of textura angularis. Conidiophores reduced to conidiogenous cells, arising from inner layers of stroma. Conidiogenous cells 3-5 $\mu \mathrm{m}$ long $\times 3-5.5$ $\mu \mathrm{m}$ wide, phialidic, integrated, short, hyaline, smooth. Conidia $16-22 \times 2.5-3.5 \mu \mathrm{m}(\bar{x}=19.5 \times 3 \mu \mathrm{m}$; $\mathrm{n}=30$ ), hyaline, subcylindrical, 1-septate, with narrow and subobtuse apex and truncate base, granular, smooth and thick-walled. 
Culture characteristics - Colonies slow growing on PDA, reaching $15 \mathrm{~mm}$ diam. after 4 weeks at $20-25^{\circ} \mathrm{C}$, circular to irregular, with uneven margins, whitened to pink, flattened, filamentous, sparse, aerial mycelium on the surface, reverse similar in colour.

Material examined - ITALY, Province of Forlì-Cesena [FC], Galeata, Passo delle Forche, on dead stem of Dactylis glomerata L. (Poaceae), 15 October 2013, Erio Camporesi, IT-719 (MFLU 150698, holotype); ex-type living culture, MFLUCC 13-0386, KUMCC 15-0134; ibid. IT-719A (KUN! HKAS 90735, isotype).

Notes - Parastagonospora allouniseptata is similar to P. uniseptata in having pycnidial, globose to subglobose, conidiomata, with hyaline, subcylindrical, 1-septate, granular conidia, but is distinct in having shorter and narrower conidia than $P$. uniseptata $(14-18 \times 2-3 \mu \mathrm{m})$. Phylogenetically, $P$. allouniseptata clusters with Parastagonospora species with $88 \%$ bootstrap support. Furthermore, $P$. allouniseptata forms a distinct branch in the Parastagonospora phylogeny (Fig. 1).

Parastagonospora dactylidis W.J. Li, Camporesi, D.J. Bhat \& K.D. Hyde, sp. nov. Fig. 4 Index Fungorum number: IF551666

Facesoffungi number: FoF 01303

Etymology - Named after the host genus, Dactylis.

Holotype - MFLU 15-0693

Saprobic on dead stem of Dactylis sp., forming numerous, conspicuous, dark brown fruiting bodies in a linear series on the surface. Sexual morph - Undetermined. Asexual morph Coelomycetous. Conidiomata 50-100 $\mu \mathrm{m}$ high, 100-150 $\mu \mathrm{m}$ diam., pycnidial, brown, separate, ampulliform or globose, immersed, unilocular, thick-walled, ostiolate. Wall of conidiomata 10-15 $\mu \mathrm{m}$ wide, composed of 3-4 layers, with outer 1-2-layers of dark brown and inner 1-2-layers of hyaline cells of textura angularis. Ostiole circular, papillate. Conidiophores reduced to conidiogenous cells. Conidiogenous cells 2-6 $\mu \mathrm{m}$ long $\times 3-8 \mu \mathrm{m}$ wide, phialidic, ampuliform, determinate, hyaline, unbranched, and developing only at the base, formed from the inner cells of the pycnidial wall. Conidia 25-40 $\times 4-5.5 \mu \mathrm{m}(\bar{x}=30 \times 4.5 \mu \mathrm{m} ; \mathrm{n}=30)$, hyaline, fusiform, curved, rounded at both ends, slightly narrower at the base, 3 -septate, constricted at the septa, with distinctly granular cytoplasm.

Culture characteristics - Colonies on PDA, reaching $20 \mathrm{~mm}$ after $2 \mathrm{wk}$ at $20-25^{\circ} \mathrm{C}$, with dense, white, flat, aerial mycelium, with rounded, smooth, margins, reverse similar in colour.

Material examined - ITALY, Province of Arezzo [AR], Passo della Consuma, on dead stem of Dactylis sp., 19 June 2012, Erio Camporesi, IT-448 (MFLU 15-0693, holotype); ex-type living culture, MFLUCC 13-0375, ICMP 20774, KUMCC15-0131; ibid. (KUN! HKAS 90738, isotype).

Notes - Parastagonospora dactylidis is most closely related to P. minima (Fig. 1). However, the individual sequence of $P$. dactylidis as compared with $P$. dactylidis has seven different substitions in the LSU sequence data. Morphologically, these two species also can be easily distinguished in form of conidia. $P$. dactylidis has fusiform conidia with a slightly narrower base, and distinctly granular cytoplasm, whereas $P$. minima has subcylindrical conidia which are wider in the basal half, and narrow at the apex. In addition, the conidiomata and conidia of $P$. dactylidis are larger and longer than those of $P$. minima (conidiomata: $40-70 \mu \mathrm{m}$ high, 50-100 $\mu \mathrm{m}$ diam., conidia: $20-28 \times 3.5-4.5$ $\mu \mathrm{m})$. Thus $P$. dactylidis is introduced as a novel species.

Parastagonospora minima W.J. Li, Camporesi, D.J. Bhat \& K.D. Hyde, sp. nov.

Fig. 5 Index Fungorum number: IF551667

Facesoffungi number: FoF 01304

Etymology - Named after the small conidiomata.

Holotype - MFLU 15-0694 

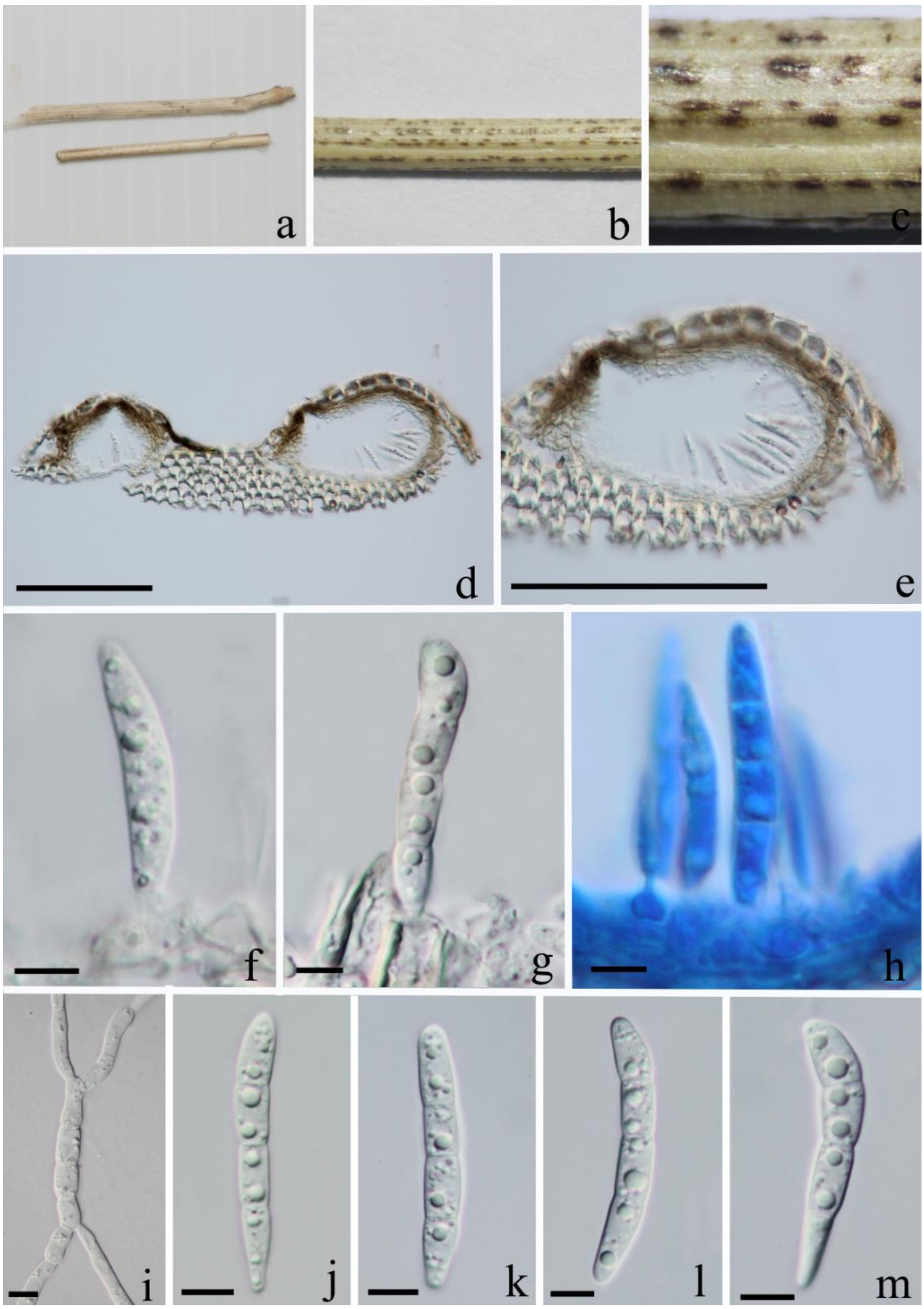

Fig. 4 - Parastagonospora dactylidis (MFLU 15-0693, holotype) a Herbarium specimen. b, c Appearance of brown conidiomata on the host. $\mathrm{d}-\mathrm{e}$ Vertical section of conidioma. $\mathrm{f}-\mathrm{h}$ Conidiogenous cells and developing conidia. i Germinating spore. $\mathrm{j}-\mathrm{m}$ Conidia. - Bars: $\mathrm{d}-\mathrm{e}=50 \mu \mathrm{m}$; $\mathrm{f}-\mathrm{m}=5 \mu \mathrm{m}$. 


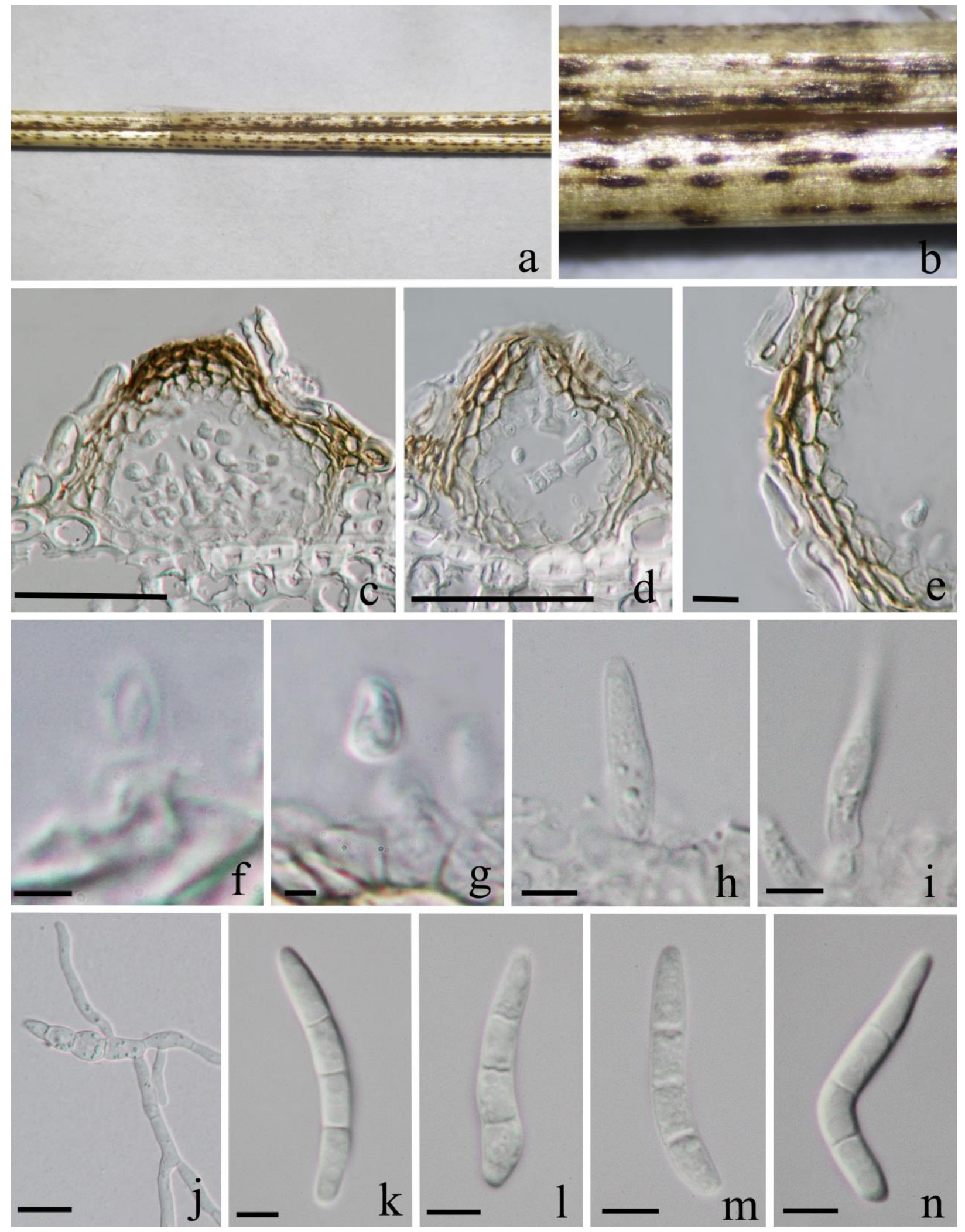

Fig. 5 - Parastagonospora minima (MFLU 15-0694, holotype) a Herbarium specimen. b Appearance of black conidiomata on the host. $c-d$ Vertical section of conidioma. e Section of peridium. $f-i$ Conidiogenous cells and developing conidia. $\mathrm{j}$ Germinating spore. $\mathrm{k}-\mathrm{n}$ Conidia. $-\mathrm{Bars}$ : $\mathrm{c}-\mathrm{d}=100 \mu \mathrm{m}$; $\mathrm{e}=20 \mu \mathrm{m} ; \mathrm{f}-\mathrm{i}, \mathrm{k}-\mathrm{m}=5 \mu \mathrm{m} ; \mathrm{j}=20 \mu \mathrm{m}$. 
Saprobic on dead stem of Dactylis sp., with dark brown fruiting bodies, in a linear series. Sexual morph - Undetermined. Asexual morph - Coelomycetous. Conidiomata 40-70 $\mu \mathrm{m}$ high, 50-100 $\mu \mathrm{m}$ diam., pycnidial, brown to black, amphigenous, separate, gregarious or confluent, obpyriform or globose, subepidermal, unilocular, ostiolate, thick-walled. Wall of conidiomata 6-10 $\mu \mathrm{m}$ wide, composed of dark brown to pale brown, thick-walled cells of textura angularis. Ostiole centrally located, papillate, circular. Conidiophores reduced to conidiogenous cells. Conidiogenous cells 3-6.5 $\mu \mathrm{m}$ long $\times 3-7 \mu \mathrm{m}$ wide, phialidic, hyaline, smooth. Conidia 20-28 $\times 3.5-4.5 \mu \mathrm{m}(\bar{x}=$ $25.5 \times 4 \mu \mathrm{m} ; \mathrm{n}=30$ ), hyaline, subcylindrical, wider at the basal half, narrow and rounded at both ends, slightly curved, 3-euseptate, smooth-walled.

Culture characteristics - Colonies on PDA, reaching $25 \mathrm{~mm}$ after 2 wk at $20-25^{\circ} \mathrm{C}$, with moderate aerial mycelium, flat, dense, spreading, with rounded, smooth margins.

Material examined - ITALY, Province of Arezzo [AR], Passo della Consuma, on dead stem of Dactylis sp., 19 June 2012, Erio Camporesi IT-451 (MFLU 15-0694, epitype); ex-type living culture, MFLUCC 13-0376, ICMP 20776, KUMCC15-0132; ibid. (KUN! HKAS 90734, isotype).

Notes - Parastagonospora minima is morphologically similar with the generic type, $P$. nodorum, in having subcylindrical, hyaline conidia, with an obtuse apex, as well as being of similar size $(P$. nodorum 13-28 × 2.8-4.6 $\mu \mathrm{m}$ ) (Quaedvlieg et al 2013). However, recognizable differences between these two species are in the septation. Parastagonospora minima has uniformly 3-septate conidia, whereas $P$. nodorum has (0-)1(-3)-septate conidia. Phylogenetically, $P$. minima is distinct from any other species of Parastagonospora (Fig.1). Based on the above morphological characters together with molecular sequence data, $P$. minima is introduced as a new species.

Parastagonospora italica W.J. Li, Camporesi, D.J. Bhat \& K.D. Hyde, sp. nov.

Fig. 6 Index Fungorum number: IF551668

Facesoffungi number: FoF 01305

Etymology - Named after the country of collection.

Holotype - MFLU 15-0696

Saprobic on dead stem of Dactylis sp.. Sexual morph - Undetermined. Asexual morph Coelomycetous. Conidiomata 65-80 $\mu \mathrm{m}$ high, (40-) 100-150 $\mu \mathrm{m}$ diam., pycnidial, brown to black, solitary or gregarious, subepidermal, immersed, unilocular, globose to subglobose, thick-walled, black. Wall of conidiomata 10-20 $\mu \mathrm{m}$ wide, composed of 2-4 layers, thick-walled cells of textura angularis, brown. Ostiole centrally located, circular, short, papillate. Conidiophores reduced to conidiogenous cells, arising from inner layers of pycnidia. Conidiogenous cells short, broadly cylindrical, phialidic, hyaline, smooth. Conidia 25-32 × 3-4 $\mu \mathrm{m}(\bar{x}=29.5 \times 3-5 \mu \mathrm{m} ; \mathrm{n}=20)$, hyaline, cylindric-fusiform, with narrow and obtuse apex, truncate at base, slightly curved, 3-euseptate, smooth and thick-walled.

Culture characteristics - Colonies on PDA, reaching $20 \mathrm{~mm}$ after $10 \mathrm{~d}$ at $20-25^{\circ} \mathrm{C}$, with crenate, white margins, pink, flattened, with dense, aerial mycelium on the surface; reverse pale-yellowish to pale brown in the middle, whitened at the margins.

Material examined - ITALY, Province of Forlì-Cesena [FC], Santa Sofia, Campigna, on dead stem of Dactylis sp., 24 June 2012, Erio Camporesi, IT-471 (MFLU 15-0696, holotype); ex-type living culture, MFLUCC 13-0377, ICMP 20777, KUMCC. ibid. 15 May 2013, Erio Camporesi, IT471A (KUN! HKAS 90736, isotype).

Notes - In the multi-locus phylogeny, Parastagonospora italica clusters with $P$. caricis Quaedvl. et al., but this grouping is not well-supported. Morphologically, these two species can easily be distinguished by dimension and septation of conidia; $P$. italica has 3-septate conidia which are shorter and narrower than the 7-15-septate, (50-)60-70(-75) $\times(5-) 6 \mu \mathrm{m}$ conidia in $P$. caricis. In addition, the conidiomata of $P$. italica are smaller than those of $P$. caricis which are up to $250 \mu \mathrm{m}$. 

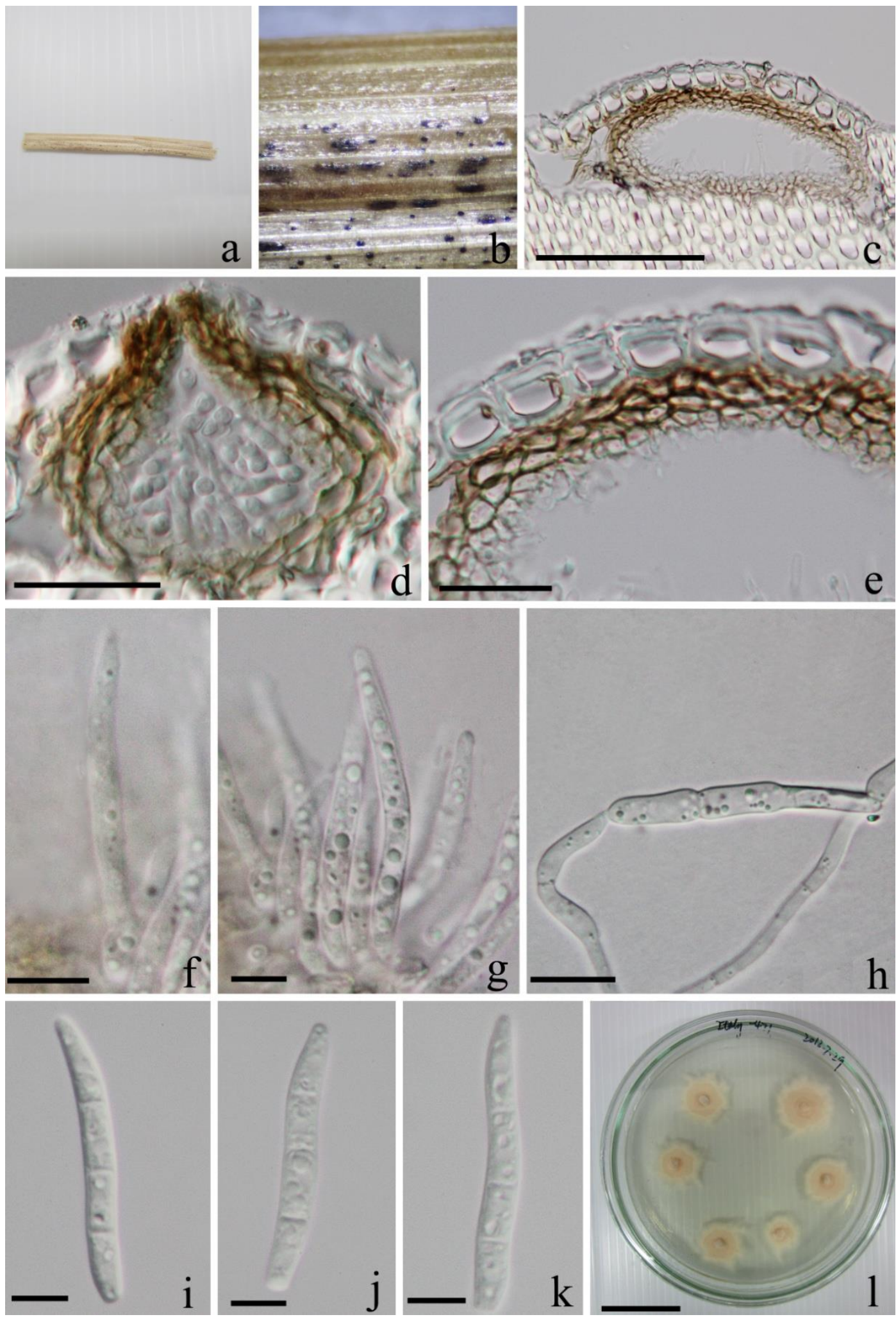

Fig. 6 - Parastagonospora italica (MFLU 15-0696, holotype) a Herbarium specimen. b Appearance of black conidiomata on the host surface. $\mathrm{c}-\mathrm{d}$ Vertical section of conidioma. e Section of peridium. $\mathrm{f}-\mathrm{g}$ Conidiogenous cells and developing conidia. h Germinating spore. i-k Conidia. 1 Culture on PDA. Bars: $\mathrm{c}=100 \mu \mathrm{m} ; \mathrm{d}=25 \mu \mathrm{m} ; \mathrm{e}=20 \mu \mathrm{m} ; \mathrm{f}-\mathrm{g}=5 \mu \mathrm{m} ; \mathrm{h}=10 \mu \mathrm{m} ; \mathrm{i}-\mathrm{k}=5 \mu \mathrm{m} ; \mathrm{l}=25 \mathrm{~mm}$. 
Parastagonospora uniseptata W.J. Li, Camporesi, D.J. Bhat \& K.D. Hyde, sp. nov.

Index Fungorum number: IF551669

Facesoffungi number: FoF 01306

Etymology - Named after the fact that conidia are 1-septate.

Holotype - MFLU 15-0699

Saprobic on dead stem of Daucus sp., forming conspicuous, to oval, black, fruiting bodies. Sexual morph - Undetermined. Asexual morph - Coelomycetous. Conidiomata 60-100 $\mu \mathrm{m}$ high, 70-100 $\mu \mathrm{m}$ diam, pycnidial, brown to black, appearing on upper surface of the stem, solitary, sometimes gregarious or confluent, immersed to semi-immersed, globose or subglobose, unilocular, with a blackened tip above, ostiolate at the centre, thick-walled. Wall of conidiomata 10-20 $\mu \mathrm{m}$ wide, composed of 6-layers, outer 4-5-layers brown, inner 2-3-layers hyaline, upper wall region dark brown, with periclinal and basal wall composed of thick-walled cells of textura angularis. Conidiophores reduced to conidiogenous cells, arising all around the basal region of the conidiomata. Conidiogenous cells 3-6 $\mu \mathrm{m}$ long $\times 3-6.5 \mu \mathrm{m}$ wide, ampulliform to broadly conical, phialidic, hyaline, smooth. Conidia $14-18 \times 2-3 \mu \mathrm{m}(\bar{x}=16 \times 2.5 ; \mathrm{n}=30)$, hyaline, subcylindrical, 1-septate, guttulate, truncate at the base, with obtuse apex, smooth-walled.

Culture characteristics - Colonies slow growing on PDA, reaching $20 \mathrm{~mm}$ diam. after 4 weeks at $20-25^{\circ} \mathrm{C}$, with a glabrous, circular margin, pale yellow, flattened, filamentous, sparse, with aerial mycelium on the surface, similar in colour from below.

Material examined - ITALY, Province of Arezzo [AR], Modigliana, Bagno di Cetica, on dead stems of Daucus sp., 18 September 2012, Erio Camporesi, IT-727 (MFLU 15-0699, holotype); extype living culture, MFLUCC 13-0387, KUMCC 15-0134; ibid. IT-727A (KUN! HKAS 90730, isotype).

Notes - Parastagonospora uniseptata should be morphologically compared with stagonosporalike asexual morphs. The results show that $P$. uniseptata is more similar to the type of Neostagonaspora, N. caricis Quaedvl. et al. than to other species within Parastogonospora. However, phylogenetic tree reconstruction based on multigene (LSU and ITS) sequence analyses shows that $P$. uniseptata clusters together with type $P$. nodorum, as well as other species of Parastagonospora $(\mathrm{BS}=$ $88 \%$ ) (Fig. 1). Parastogonospora uniseptata however, forms a discrete branch basal to P. poae Quaedvl.et al. (Fig. 1). Thus P. uniseptata is introduced as a new species in Parastagonospora.

Poaceicola W.J. Li, Camporesi, D.J. Bhat \& K.D. Hyde, gen. nov. Index Fungorum number: IF551658

Facesoffungi number: FoF 01298

Etymology - Named after inhabiting grass (Poaceae).

Type species - Poaceicola arundinis W.J. Li, Camporesi, D.J. Bhat \& K.D. Hyde, sp. nov.

Saprobic on dead stems of plant host. Sexual morph - Ascomata solitary to gregarious, black, immersed to semi-immersed, subepidermal, uniloculate, glabrous, globose to subglobose, papillate. Ostiole centrally located, black, smooth, with an ostiole, filled with hyaline periphyses. Peridium thick-walled, brown, composed of cells of textura angularis. Hamathecium comprising cellular, septate, broad, dense pseudoparaphyses. Asci 8-spored, bitunicate, fissitunicate, cylindrical, with a short, broad pedicel. Ascospores tetraseriate or partially overlapping, reddish-brown, fusiform, straight or inequilaterally curved, echinulate, 10-septate, fourth cell from apex swollen towards middle and slightly longer than adjacent cells, with a conspicuous sharply delimited sheath. Asexual morph Coelomycetous. Conidiomata pycnidioid, solitary or aggregated, immersed or semi-immersed, unilocular, globose, papillate, dark brown. Wall of conidiomata comprising inner layers of textura angularis cells, gradually merging with the outer surrounding layers of brown, textura oblita. Ostiole 

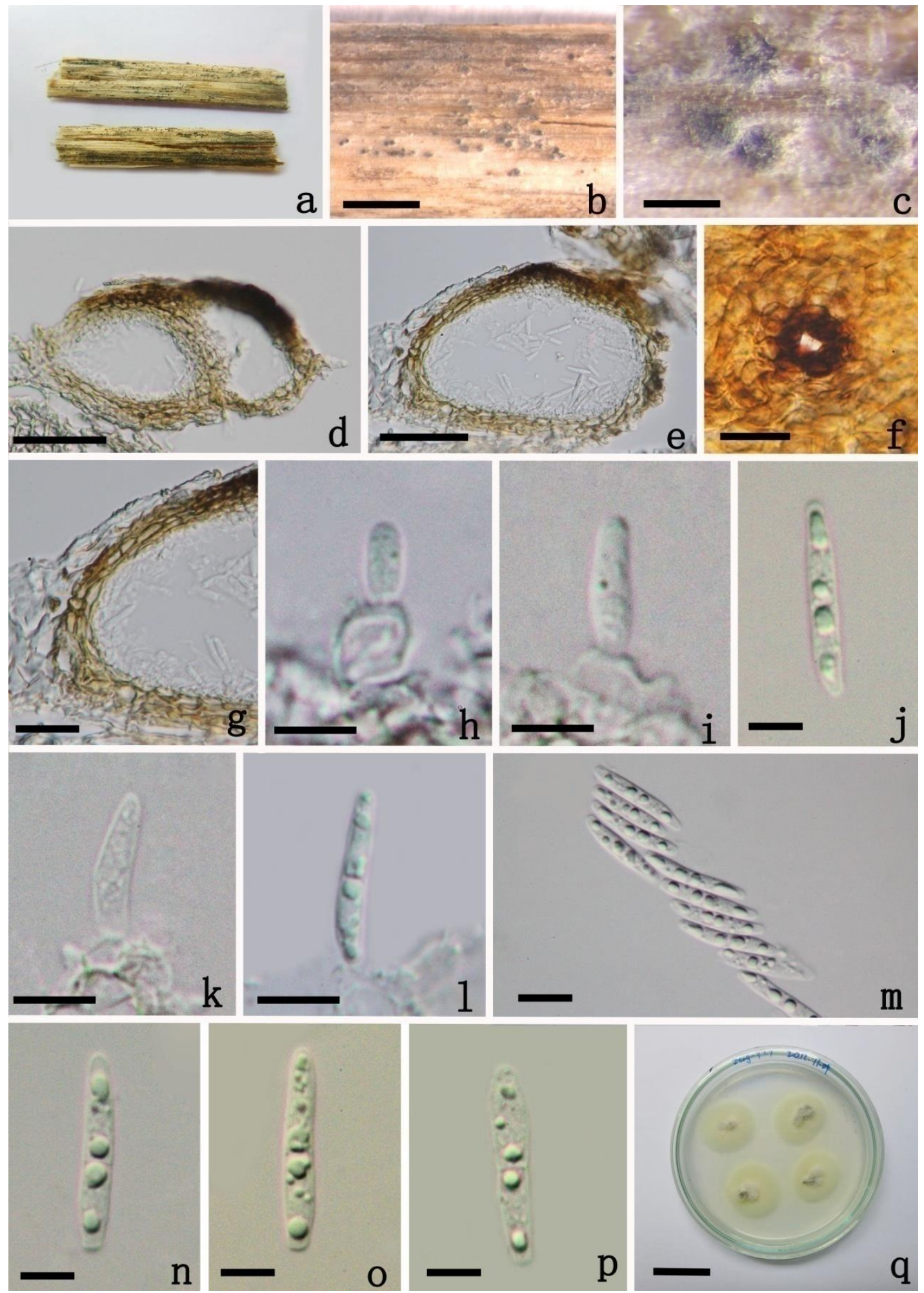

Fig. 7 - Parastagonospora uniseptata (MFLU 15-0699, holotype) a Herbarium specimen. b-c Appearance of black conidiomata on the host. d-e Vertical section of conidiomata. f Ostiole. g Section of peridium. $\mathrm{h}-\mathrm{i}, \mathrm{k}-\mathrm{l}$ Conidiogenous cells and developing conidia. $\mathrm{j}, \mathrm{m}-\mathrm{p}$ Conidia. q Culture on PDA. - Bars: $\mathrm{b}-\mathrm{c}=200 \mu \mathrm{m} ; \mathrm{d}-\mathrm{e}=50 \mu \mathrm{m} ; \mathrm{f}=15 \mu \mathrm{m} ; \mathrm{g}=20 \mu \mathrm{m} ; \mathrm{h}-\mathrm{i}, \mathrm{k}-\mathrm{l}=5 \mu \mathrm{m} ; \mathrm{j}, \mathrm{m}-\mathrm{p}=5 \mu \mathrm{m} ; \mathrm{q}=25$ $\mathrm{mm}$. 
central, circular to oval, papillate. Conidiophores reduced to conidiogenous cells. Conidiogenous cells holoblastic, phialidic, hyaline, smooth-walled, discrete, formed from the inner cells of the stroma. Conidia pale brown, cylindrical or sub-cylindrical, up to 7-euseptate, straight or slightly curved, smooth-walled,.

Notes - The phylogeny of the family Phaeosphaeriaceae is reconstructed based on combined gene (LSU and ITS) analysis, showing that Poaceicola species cluster away from any other genera in Phaeosphaeriaceae (Fig. 1). Thus the genus Poaceicola is introduced to accommodate Poaceicola elongata comb. nov. and two asexual morphs introduced in this study i.e. Po. arundinis sp. nov. and Po. bromi sp. nov.

Poaceicola arundinis W.J. Li, Camporesi, D.J. Bhat \& K.D. Hyde, sp. nov. Fig. 8 Index Fungorum number: IF551659

Facesoffunginumber: FoF 01299

Etymology - Named after the host genus Arundo.

Holotype - MFLU 15-0702

Saprobic on dead stems of Arundo plinii Turra, forming conspicuous, rounded, black fruiting bodies. Sexual morph - Undetermined. Asexual morph - Coelomycetous. Conidiomata 100-150 $\mu \mathrm{m}$ high, 100-200 $\mu \mathrm{m}$ diam., pycnidioid, dark brown, solitary or aggregated, semi-immersed, unilocular, globose, papillate. Wall of conidiomata 10-30 $\mu \mathrm{m}$ wide, comprising inner layers of cells of textura angularis, gradually merging with the outer, surrounding layers of brown, textura oblita. Ostiole central, circular to oval, papillate. Conidiophores reduced to conidiogenous cells. Conidiogenous cells 3-6 $\mu \mathrm{m}$ wide, holoblastic, phialidic, hyaline, smooth-walled, discrete, formed from the inner cells of the stroma. Conidia 30-40 $\times 6.5-10 \mu \mathrm{m}(\bar{x}=35 \times 8 \mu \mathrm{m} ; \mathrm{n}=30)$, pale brown, cylindrical, flexuous, up to 8-euseptate, slightly curved, smooth-walled, with middle cells wider than end cells, guttulate, with an acute apex, truncate at the base.

Material examined - ITALY, Province of Arezzo [AR], Montemezzano, on dead on dead stems of Arundo plinii Turra, 25 August 2013, Erio Camporesi, IT-938 (MFLU 15-0702); ibid. (KUN! HKAS 90732).

Notes - Poaceicola arundinis is distinct from Po. bromi in conidiomata and conidia form. Poaceicola arundinis has globose conidiomata with circular to oval, short ostioles, while Po. bromi has pyriform conidiomata with cylindrical, long ostioles. In addition, the conidia of Po. arundinis with up to 8-septa are longer and wider than those of Po. bromi which are 7-septate and 15-23 $\times 3-5 \mu \mathrm{m}$.

Poaceicola bromi Wijayawardene, W.J. Li, Camporesi, D.J. Bhat \& K.D. Hyde, sp. nov.

Fig. 9 Index Fungorum number: IF551660

Facesoffungi number: FoF 01300

Etymology - Named after the host genus, Bromus.

Holotype - MFLU 15-2719

Saprobic on dead twigs of Bromus sterilis L. (Poaceae). Sexual morph - Undetermined. Asexual morph - Coelomycetous. Conidiomata 310-350 $\mu \mathrm{m}$ high, 240-280 $\mu \mathrm{m}$ diam., pycnidioid, dark brown, solitary, semi-immersed, unilocular, globose, papillate. Wall of conidiomata 29-49 $\mu \mathrm{m}$ wide, thick-walled, composed of thick-walled cells of textura angularis at the outside, with inner layers lighter and flattened. Setae 20-30 × 5-6 $\mu \mathrm{m}$, lateral, sparse, subcylindrical, 1-2-septate, dark brown. Ostiole centrally located, cylindrical to subcylindrical, papillate. Conidiophores reduced to conidiogenous cells. Conidiogenous cells 1-2 × 1-3 $\mu \mathrm{m}$, holoblastic, phialidic, hyaline, smoothwalled, discrete, formed from the inner cells of the stroma. Conidia 15-23 $\times 3-5 \mu \mathrm{m}(\bar{x}=20.5 \times 4$ $\mu \mathrm{m} ; \mathrm{n}=30$ ), pale brown to dark brown, cylindrical, straight or slightly curved, obtuse at both ends, up to 7-euseptate, constricted at the septa, smooth-walled. 

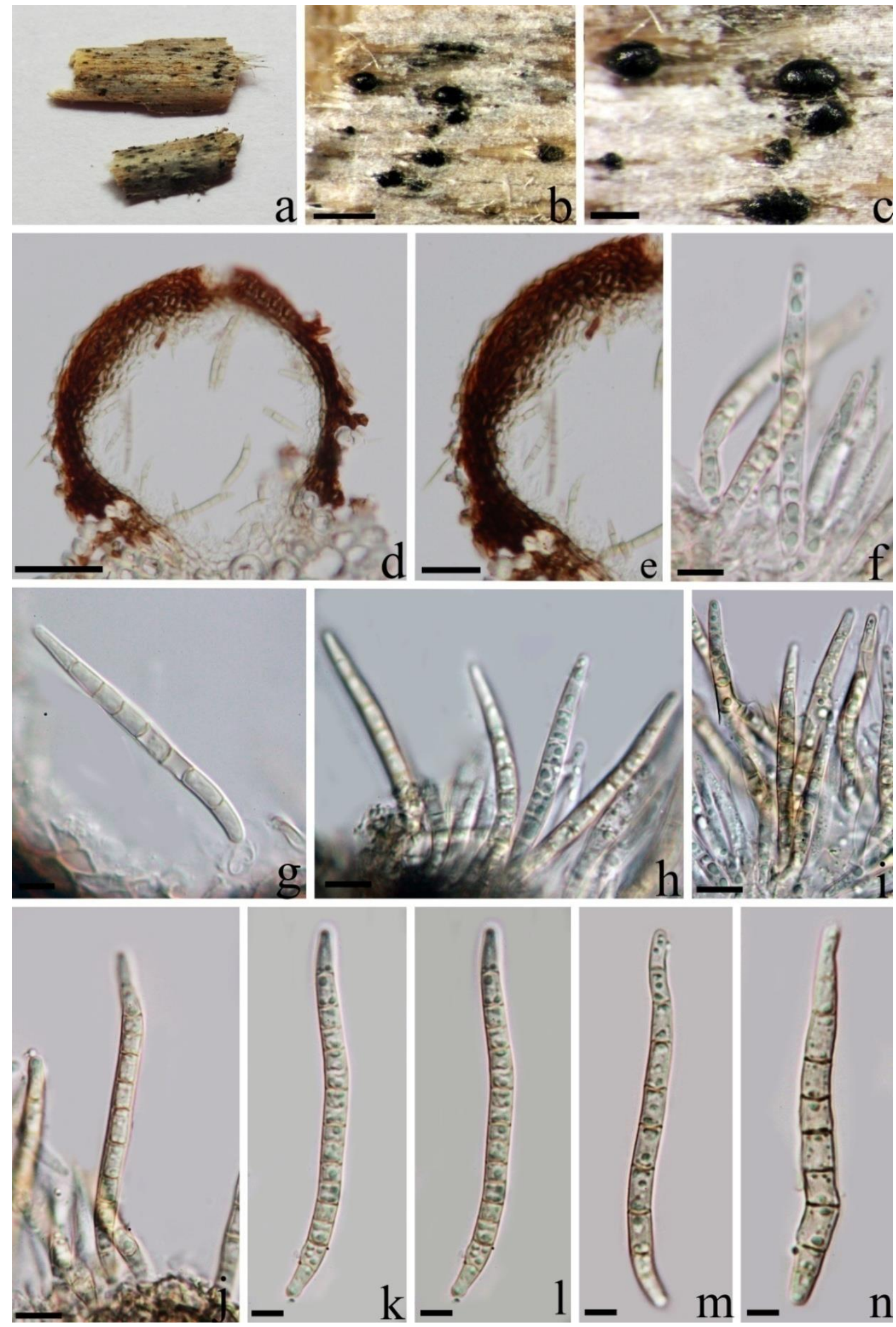

Fig. 8 - Poaceicola arundinis (MFLU 15-0702, holotype) a Herbarium specimen. b-c Appearance of black conidiomata on the host. d Vertical section of conidioma. e Section of peridium. f-i Conidiogenous cells and developing conidia. $\mathrm{j}-\mathrm{n}$ Conidia. $\mathrm{m}$ Germinating spore. $\mathrm{n}$ Culture on PDA. Bars: $\mathrm{b}=200 \mu \mathrm{m} ; \mathrm{c}=20 \mu \mathrm{m} ; \mathrm{d}=100 \mu \mathrm{m} ; \mathrm{e}=50 \mu \mathrm{m} ; \mathrm{f}-\mathrm{i}=10 \mu \mathrm{m} ; \mathrm{j}-\mathrm{n}=10 \mu \mathrm{m}$. 

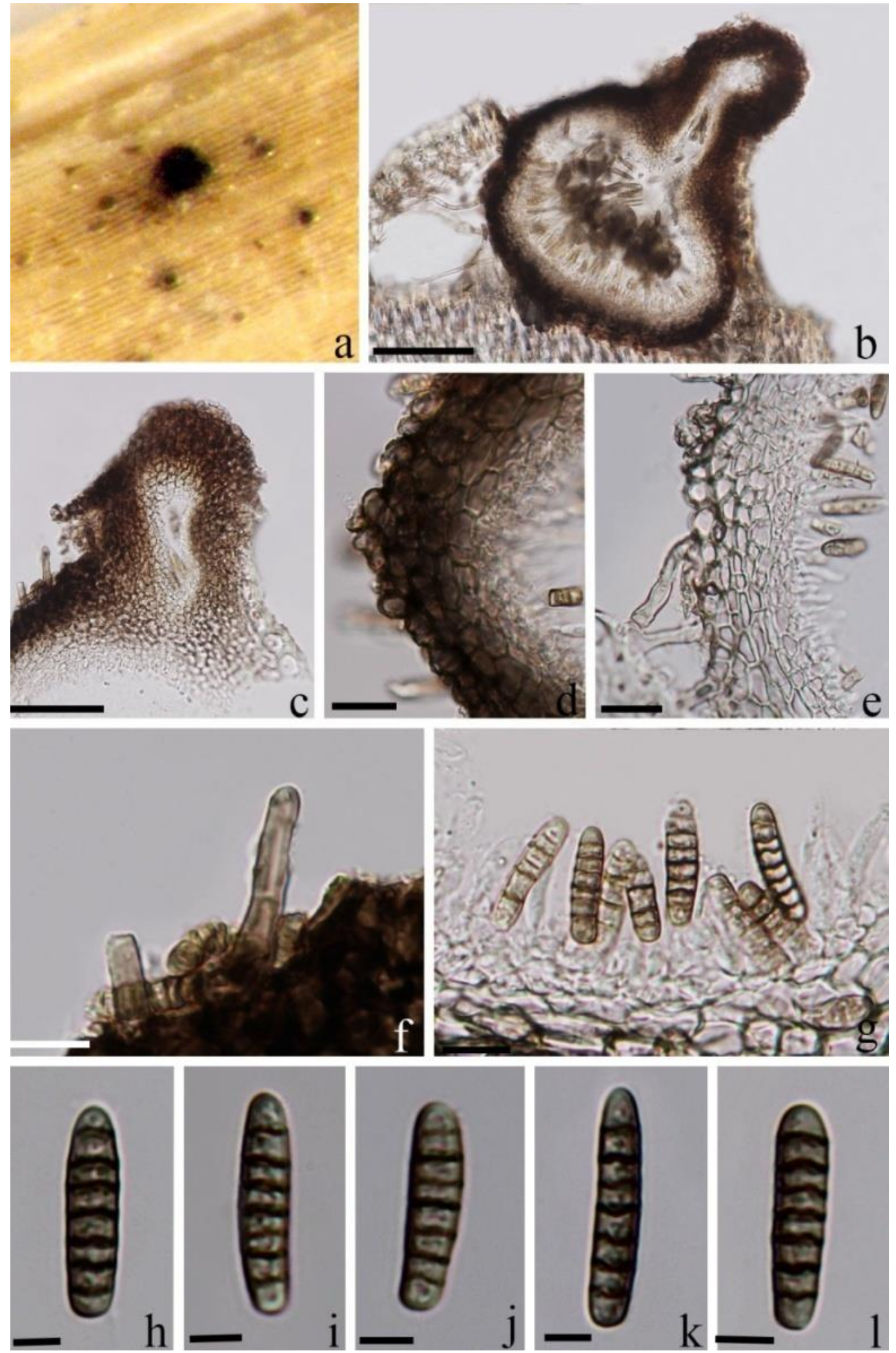

Fig. 9 - Poaceicola bromi (MFLU MFLU 15-2719, holotype) a Appearance of black conidioma on host. b Vertical section of conidioma. c Ostiole. $\mathrm{d}-\mathrm{e}$ Section of peridium. $\mathrm{f}-\mathrm{g}$ Conidiogenous cells and developing conidia. $\mathrm{h}-1$ Conidia. - Bars: $\mathrm{b}=100 \mu \mathrm{m} ; \mathrm{c}=50 \mu \mathrm{m} ; \mathrm{d}-\mathrm{e}=20 \mu \mathrm{m} ; \mathrm{f}-\mathrm{g}=10 \mu \mathrm{m} ; \mathrm{h}-1=5$ $\mu \mathrm{m}$. 
Culture characteristics - On PDA slow growing, attaining $2 \mathrm{~cm}$ diam. in 7 days, with circular to slightly wavy margin, greyish white from above, grey from below, with thin mycelium.

Material examined - ITALY, Province of Forlì-Cesena [FC], on leaves of Bromus sterilis L. (Poaceae), 19 July 2013, Erio Camporesi, NNW IT-1389 (MFLU 15-2719, holotype). (KUN! HKAS 90729, isotype), living cultures, MFLUCC 13-0739, GUCC 1389

Notes - Poaceicola bromi is morphologically and phylogenetically distinct from Po. arundinis; see notes under Po. arundinis.

Poaceicola elongata (Wehm.) Shoemaker \& C.E. Babc.) W.J. Li, Camporesi \& K.D. Hyde, comb. nov.

Index Fungorum number: IF551661

Facesoffungi number: FoF 01301

Holotype - MFLU14-0635

Basionym - Phaeosphaeria elongata (Wehm.) Shoemaker \& C.E. Babc., Can. J. Bot. 67(5): 1540 (1989)

Synonym - Leptosphaeria elongata Wehm., Mycologia 44: 633 (1952). 633 (1952).

Notes - This specimen was collected from dead wood in Italy. Ariyawansa et al. (2014) designated the collection as a reference specimen of Phaeosphaeria elongata. However, P. elongata clusters close to Po. cylindrispora and Po. bromi with high bootstrap support (BS =96\%) (Fig. 1), and is best assigned to Poaceicola.

Septoriella allojunci W.J. Li, Camporesi, D.J. Bhat \& K.D. Hyde, sp. nov.

Fig. 10

Index Fungorum number: IF551662

Facesoffungi number: FoF 01308

Etymology - Named after its morphological similar to Septoriella junci.

Holotype - MFLU 15-0701

Saprobic on dead stem of Juncus sp.. Sexual morph - Undetermined. Asexual morph Coelomycetous. Conidiomata 90-130 $\mu \mathrm{m}$ high, 100-150 $\mu \mathrm{m}$ diam., stromatic, pycnidial, dark brown, solitary or aggregated, linearly disposed with only the dark spots visible in surface view, immersed, unilocular, globose, papillate. Ostiole centrally located, circular to oval, apapillate. Wall of conidiomata 10-20 $\mu \mathrm{m}$ wide, composed of brown, thick-walled cells of textura angularis, at the base, thin-walled, dark brown cells in upper part and surrounding the ostiole. Conidiophores reduced to conidiogenous cells. Conidiogenous cells 3-6 $\mu \mathrm{m}$ wide, holoblastic, discrete, phialidic, ampulliform, hyaline, smooth-walled, formed from the inner cells of the conidiomata. Conidia 48-70 $\times 3-6.6 \mu \mathrm{m}$ $(\bar{x}=60 \times 4 \mu \mathrm{m} ; \mathrm{n}=30)$, pale brown, subcylindrical, flexuous, with obtuse ends, 3-5-euseptate, smooth-walled, guttulate, with mucoid cap at the apex.

Material examined - ITALY, Province of Forlì-Cesena [FC], Galeata, Strada San Zeno, on dead stems of Juncus sp. (Juncaceae), 27 November 2012, Erio Camporesi, IT-930 (MFLU 15-0701, holotype); ibid. (KUN! HKAS 90733, isotype).

Notes - Crous et al. (2015b) fixed the application of the type species of generic name (Septoriella phragmitis Oudem.), and confirmed the placement of Septoriella in Phaeosphaeriaceae. In the present study, we introduced an additional taxon, Septoriella allojunci. Morphologically, $S$. allojunci shows similarities with $S$. junci and $S$. canadensis in the form of conidiomata and conidiogenous cells, but differs in the dimension, and septation of conidia. Septoriella allojunci has 35-septate conidia which are longer and wider than those of $S$. canadenesis which have 3-4-septate conidia $(36-56 \times 2.5-3.5 \mu \mathrm{m})$, as well as shorter and wider than $S$. junci (6-7-septate, 49-90 $\times 2-3$ $\mu \mathrm{m})$. Phylogenetically, $S$. allojunci is close to $S$. phragmitis (Fig. 1). 

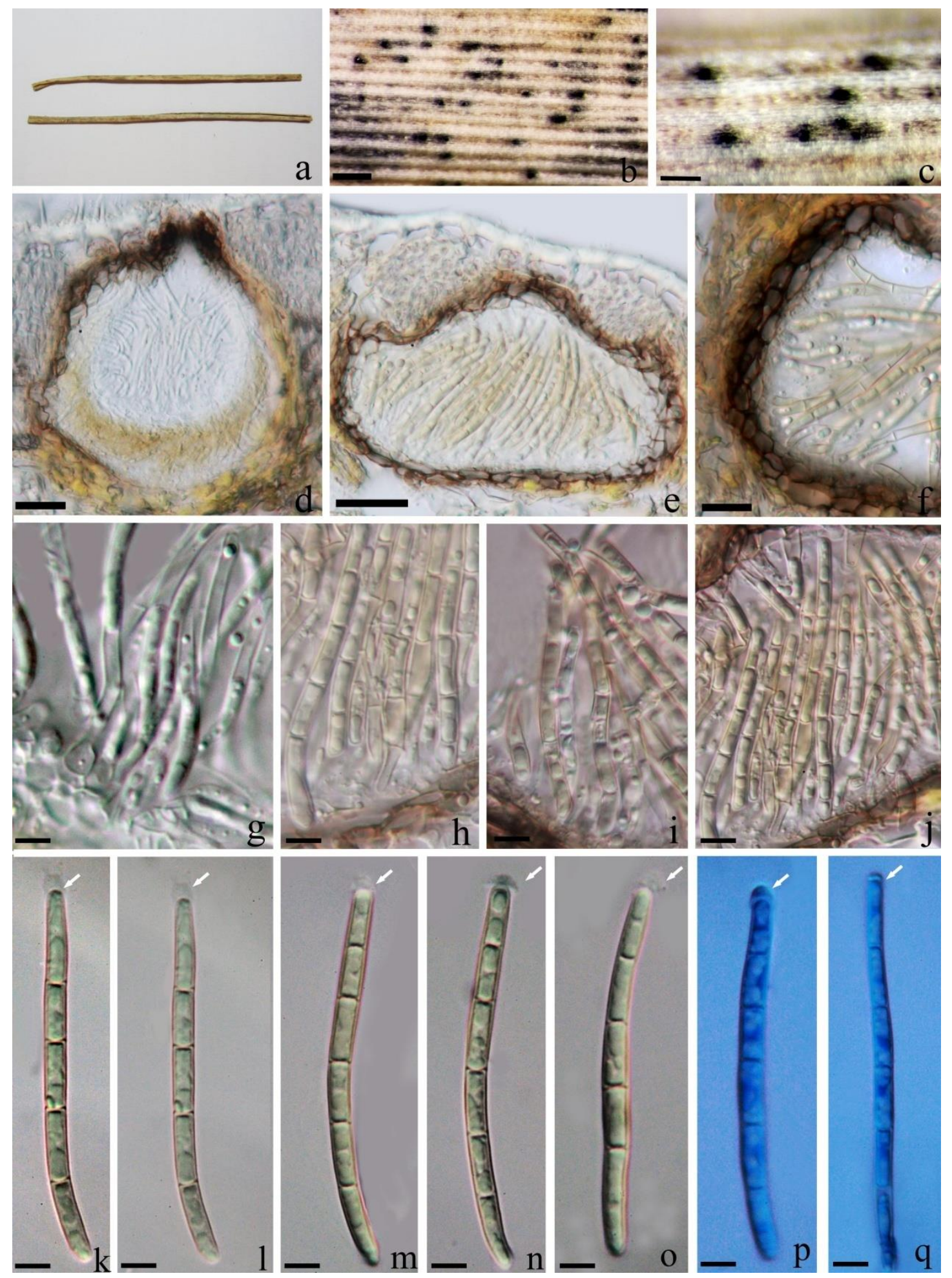

Fig. 10 - Septoriella allojunci (MFLU 15-0701, holotype) a Herbarium specimen. b-c Appearance of black conidiomata on the host surface. $\mathrm{d}-\mathrm{e}$ Vertical section of conidioma. f Section of conidioma. $\mathrm{g}-\mathrm{j}$ Conidiogenous cells and developing conidia. $\mathrm{k}-\mathrm{q}$ Conidia. - Bars: $\mathrm{b}-\mathrm{c}=200 \mu \mathrm{m} ; \mathrm{d}-\mathrm{e}=100 \mu \mathrm{m} ; \mathrm{f}=$ $50 \mu \mathrm{m} ; \mathrm{g}-\mathrm{j}=10 \mu \mathrm{m} ; \mathrm{k}-\mathrm{q}=10 \mu \mathrm{m}$. 
Wojnowicia spartii W.J. Li, Camporesi \& K.D. Hyde, sp. nov.

Index Fungorum number: IF551663

Facesoffungi number: FoF 01309

Etymology - Named after the host genus, Spartium.

Holotype - MFLU 15-0700

Saprobic on dead stem of Spartium sp., forming conspicuous, round, black fruiting bodies. Sexual morph - Undetermined. Asexual morph - Coelomycetous. Conidiomata 120-180 $\mu \mathrm{m}$ high, 140-200 $\mu \mathrm{m}$ diam., pycnidial, dark brown, scattered, immersed to semi-immersed, unilocular, globose to subglobose, glabrous, ostiolate. Ostiole centrally located, papillate, cylindrical. Wall of conidiomata 13-28 $\mu \mathrm{m}$ wide, composed of 4-5-cell layers, of thick-walled cells of textura angularis, and inner layers of light brown to hyaline textura angularis. Conidiophores reduced to conidiogenous cells. Conidiogenous cells 2.8-6.5 $\mu \mathrm{m}$ long $\times 3-7 \mu \mathrm{m}$ wide, phiallidic, hyaline, integrated, flask-shaped, thick-walled. Conidia 28-36 $\times 5-6(\bar{x}=28 \times 5 \mu \mathrm{m} ; \mathrm{n}=30)$, dark brown, fusiform or cylindrical, straight or slightly curved, wide in the middle, 7-12-septate, constricted at septa, subobtuse at each end, thick-walled, smooth-walled, guttulate.

Culture characteristics - Colonies slow growing on PDA, reaching $10 \mathrm{~mm}$ diam. after 5d at 20$25^{\circ} \mathrm{C}$, circular, flattened, with sparse, grey to olivaceous aerial mycelium, surface patches of olivaceous-grey in the older parts; reverse olivaceous-black.

Material examined - ITALY, Province of Forlì-Cesena [FC], Bagno di Romagna, Passo del Carnaio, on dead stem of Spartium sp., 17 November 2012, Erio Camporesi, IT-889 (MFLU 15-0700, holotype); ex-type living culture, MFLUCC 13-0402, ICMP 20790, KUMCC 15-0136; ibid. (KUN! HKAS 90737, isotype).

Notes - Wojnowicia was introduced by Saccardo (1892) with W. hirta as the type species. Subsequently, the genus was expanded to include two more species, viz. W. colluvium D.F. Farr \& Bills and W. ephedrae Hollós (Sutton 1980, Farr \& Bills 1995). According to Index Fungorum (2015) there are 14 species epithets in Wojnowicia. However, W. graminis (McAlpine) Sacc. \& D. Sacc. and W. tenella Pat. were synonymised under W. hirta by Sutton (1980). Wojnowicia buxi Bertault \& Malençon was synonymised under W. ephedrae Hollós by Farr \& Bills (1995). Wojnowicia bryophila Racov., W. exilis (Corda) Sacc. \& Traverso and W. lophostoma (Höhn.) Sacc. were excluded from Wojnowicia (Sutton 1980, Farr \& Bills 1995, Wijayawardene et al. 2013). Wojnowicia viburni Wijayaw. et al. was transferred to Wojnowiciella (Crous et al. 2015a). Presently, seven species are accepted in Wojnowicia, including W. spartii described in this paper (Sutton 1980, Wijayawardene et al. 2013, Liu et al. 2015).

Wojnowicia spartii should be compared with W. dactylidis Wijayaw. et al. and W. lonicerae Wijayaw.et al. However, conidia of $W$. spartii are shorter than in $W$. dactylidis $(35-40 \times 4-5.5 \mu \mathrm{m})$ and $W$. lonicerae $(38-49 \times 5-6 \mu \mathrm{m})$. Wojnowicia spartii has similar conidial dimensions as $W$. dactylidicola Wijayaw. et al. $(25-35 \times 3.5-6.5 \mu \mathrm{m}(\bar{x}=28.38 \times 4.87 \mu \mathrm{m})$, but can be distinguished using septation (7-12 septa in W. spartii and 3-5 septa in W. dactylidicola). Multigene analyses shows that W. spartii is distinct from any other Wojnowicia species (Fig. 1).

\section{Discussion}

Many genera of ascomycetes are pleomorphic and propagate through sexual or asexual reproduction in different geographical locations and at different times, and it is often difficult to understand the whole life cycle of a species (Hyde et al. 2011, Wijayawardene et al. 2012). Observation of sporulating structures from single ascospores on artificial media, the occurrence of two morphs on the same substrate, or mating studies was previously the only way to link morphs (Wijayawardene et al. 2014). However, the occurrence of an asexual morph adjacent to the sexual morph on a host cannot equivocally show they are linked; they may be unrelated species. DNA 


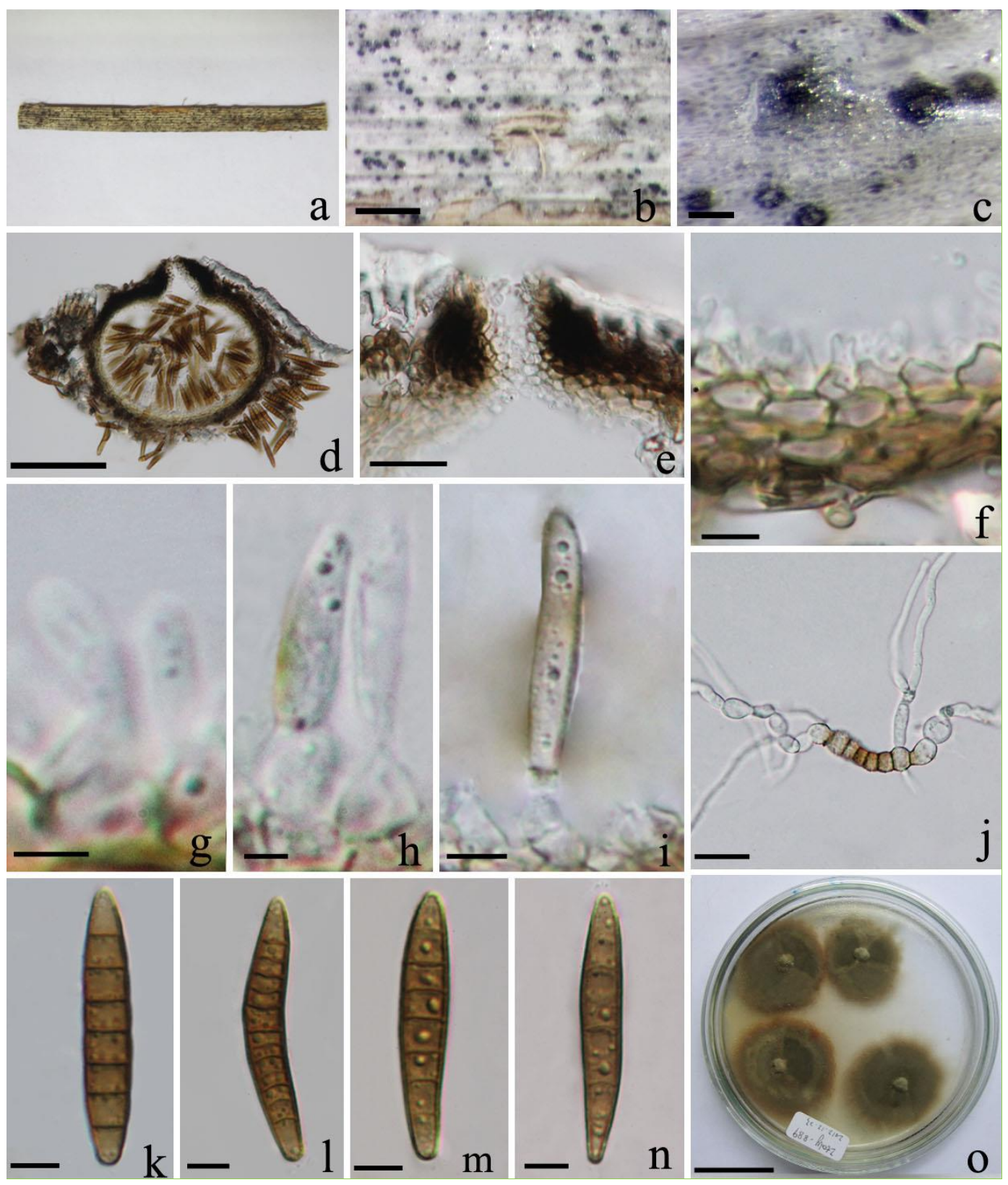

Fig. 11 - Wojnowicia spartii (MFLU 15-0700, holotype) a Herbarium specimen. b-c Appearance of black conidiomata on the host. $d$ Vertical section of conidioma. e Ostiole. f Section of peridium. $j-i$ Conidiogenous cells and developing conidia. $\mathrm{j}$ Germinating spore. $\mathrm{k}-\mathrm{n}$ Conidia. o Culture on PDA. Bars: $\mathrm{b}=200 \mu \mathrm{m} ; \mathrm{c}=200 \mu \mathrm{m} ; \mathrm{d}=100 \mu \mathrm{m} ; \mathrm{e}=20 \mu \mathrm{m} ; \mathrm{f}=20 \mu \mathrm{m} ; \mathrm{g}-\mathrm{i}=5 \mu \mathrm{m} ; \mathrm{j}-\mathrm{n}=10 \mu \mathrm{m} ; \mathrm{o}=25$ $\mathrm{nm}$.

sequence analysis and phylogenetic inference can irrefutably establish asexual and sexual morph connections, for example, the Mycosphaerella arbuticola / Septoria unedonis, Phaeosphaeria papaya / Phaeoseptoria oryzae, Leptosphaeria maculans / Plenodomus lingam, and also provide phylogenetic placements for asexual taxa within the modern taxonomic classification schemes (Quaedvlieg et al. 2013; Ariyawansa et al. 2015b, Crous et al. 2015b). In this study, sequence data together with morphology are used to delimit and propose several new species and one new genus in Phaeosphaeriaceae and link the sexual and asexual morph of Nodulosphaeria. 
The observations presented in this study show that the LSU and ITS sequence data can be used to ditinguish most genera in Phaeosphaeriaceae such as Parastagonospora and Nodulosphaeria. Nevertheless, the circumscription of Septoriella is still unclear, and our phylogenetic analysis (Fig. 1) suggests that Septoriella leuchtmannii should be excluded from the genus. Future studies should use more isolates and gene regions to resolve the genetic concepts of Septoriella.

\section{Acknowledgments}

The authors extend their sincere appreciations to the Deanship of Scientific Research at King Saud University for its funding this Prolific Research group (PRG-1436-09). We would like to thank Humidtropics, a CGIAR Research Program that aims to develop new opportunities for improved livelihoods in a sustainable environment, for partially funding this work. KD Hyde thanks The Chinese Academy of Sciences, project number 2013T2S0030, for the award of Visiting Professorship for Senior International Scientists at Kunming Institute of Botany. The authors also thank MFU Dothideomycetes grant for supporting this study. The authors would like to thank Shaun Pennycook for assistance in checking the name of new taxa.

\section{References}

Ariyawansa HA, Hawksworth DL, Hyde KD, Jones EBG, Maharachchikumbura SSN, Camporesi E, Manamgoda DS, Thambugala KM, Udayanga D, Daranagama A, Jayawardena R, Liu JK,

McKenzie EHC, Phookamsak R, Senanayake IC, Shivas RG, Tian Q, X JC. 2014 Epitypification and neotypification: guidelines with appropriate and inappropriate examples. Fungal Diversity 69, 57-91. http://dx.doi.org/10.1007/s13225-014-0315-4

Ariyawansa HA, Hyde KD, Jayasiri SC, Buyck B, Chethana KWT, Cui YY, Dai DQ, Dai YC, Daranagama DA, Jayawardena RS, Lücking R, Ghobad-Nejhad M, Niskanen T, Thambugala KM, Voigt K, Zhao RL, Boonmee S, Bahkali AH, Chen J, Cui BK, Dayarathne MC, Dissanayake AJ, Ekanayaka AH, Hashimoto A, Hongsanan S, Jones EBG, Larsson E, Lewis D, Li WJ, Li QR, Liu JK, Luo ZL, Maharachchikumbura SSN, Mapook A, McKenzie EHC, Norphanphoun C, Pang KL, Perera RH, Phookamsak R, Phukhamsakda C, Randrianjohany E, Senanayake IC, Singtripop C, Shang QJ, Tanaka K, Tian Q, Tian CM, Tibpromma S, Verbeken A, Abdel-Wahab MA, Wanasinghe D, Wijayawardene NN, Zhang JF, Zhang H, Abdel-Aziz FA, Adamčík S, Ammirati JF, Bulgakov T, Cabral AL, Callaghan TM, Callac P, Chang CH, Coca LF, Dal-Forno M, Dollhofer V, Fliegerová K, Greiner K, Griffith GW, Ho HM, Hofstetter V, Jeewon R, Kang JC, Kirk PM, Kytövuori I, Lawrey JD, Li XH, Liu ZY, Liu XZ, Liimatainen K, Lumbsch HT, Matumura M, Moncada B, Nuankaew S, Parnmen S, de A. Santiago M, Sato G, Sommai S, Song Y, de Souza CAF, de Souza-Motta CM, Su HY, Suetrong S, Wang Y, Wei SF, Wen TC, Yuan HS, Zhou LW, Reblova M, Fournier J, Camporesi E. 2015c - Fungal Diversity Notes 111-252 - Taxonomic and phylogenetic contributions to fungal taxa. Fungal Diversity (In press) http://dx.doi.org/10.1007/s13225-0150346-5

Ariyawansa HA, Thambugala KM, Manamgoda DS, Jayawardena R, Camporesi E, Boonmee S, Wanasinghe DN, Phookamsak R, Hongsanan S, Singtripop C, Chukeatirote E, Kang JC, Jones EBG, Hyde KD. 2015a - Towards a natural classification and backbone tree for Pleosporaceae. Fungal Diversity 71, 85-139.http://dx.doi.org/ 10.1007/s13225-015-0323-z

Ariyawansa HA, Phukhamsakda C, Thambugala KM, Bulgakov TS, Wanasinghe DN, Perera RH, Mapook A, Camporesi E, Kang JC, Jones EBG, Bahkali AH, Jayasiri SC, Hyde KD, Liu ZY, Bhat JD. 2015b - Revision and phylogeny of Leptosphaeriaceae. Fungal Diversity 75, (online). http://dx.doi.org/10.1007/s13225-015-0349-2 
Arzanlou M, Crous PW. 2006 - Phaeosphaeriopsis musae. Fungal Planet 9. CBS- KNAW Fungal Biodiversity Centre, Utrecht, The Netherlands.

Barr ME 1979 - A classification of Loculoascomycetes. Mycologia 71, 935-957

Chomnunti P, Hongsanan S, Aguirre-Hudson B, Tian Q, Peršoh D, Dhami MK, Alias AS, Xu JC, Liu XZ, Stadler M, Hyde KD 2014) The sooty moulds. Fungal Diversity 66,1-36.

http://dx.doi.org/10.1007/s13225-014-0278-5

Crous PW, Slippers B, Wingfield MJ, Rheeder J, Marasas WFO, Phillips AJL, Alves A, Burgess T, Barber P, Groenewald JZ 2006 - Phylogenetic lineages in the Botryosphaeriaceae. Studies in Mycology 55, 235-253. http://dx.doi.org/10.3767/003158515X685841

Crous PW, Carris LM, Giraldo A, Groenewald JZ, Hawksworth DL, Hernández-Restrepo M, Jaklitsch WM, Lebrun MH, Schumacher RK, Stielow JB, van der Linde EJ, Vilcāne J, Voglmayr H, Wood AR. 2015b - The Genera of Fungi - fixing the application of the type species of generic names - G 2: Allantophomopsis, Latorua, Macrodiplodiopsis, Macrohilum, Milospium, Protostegia, Pyricularia, Robillarda, Rotula, Septoriella, Torula and Wojnowicia. IMA Fungus 6,163-198. http://dx.doi.org/10.5598/imafungus.2015.06.01.11.

Crous PW, Wingfield MJ, Guarro J, Hernández-Restrepo M, Sutton DA, Acharya K,Barber PA, Boekhout T, Dimitrov RA, Dueñas M, Dutta AK, Gené J, Gouliamova DE, Groenewald M, Lombard L, Morozova OV, Sarkar J, Smith MT, Stchigel AM, Wiederhold NP, Alexandrova AV, Antelmi I, Armengol J, Barnes I, Cano-Lira JF, Castañeda Ruiz RF, Contu M, Courtecuisse PrR, da Silveira AL, Decock CA, de Goes A, Edathodu J, Ercole E, Firmino AC, Fourie A, Fournier J, Furtado EL, Geering ADW, Gershenzon J, Giraldo A, Gramaje D, Hammerbacher A, He XL, Haryadi D, Khemmuk W, Kovalenko AE, Krawczynski R, Laich F, Lechat C, Lopes UP, Madrid H, Malysheva EF, Marín-Felix Y, Martín MP, Mostert L, Nigro F, Pereira OL, Picillo B, Pinho DB, Popov ES, Rodas Peláez CA, Rooney-Latham S, Sandoval-Denis M, Shivas RG, Silva V, Stoilova-Disheva MM, Telleria MT, Ullah C, Unsicker SB, van der Merwe NA, Vizzini A, Wagner HG, Wong PTW, Wood AR, Groenewald JZ. 2015a - Fungal Planet description sheets: 320-370. Persoonia 34, 167-266. http://dx.doi.org/10.3767/003158515X688433

De Gruyter J, Woudenberg JHC, Aveskamp MM, Verkley GJM, Groenewald JZ, Crous PW 2010 Systematic reappraisal of species in Phoma section Paraphoma, Pyrenochaeta \& Pleurophoma. Mycologia 102, 1066-1081. http://dx.doi.org/10.3852/09-240

Farr DF, Bills GF. 1995 - Wojnowicia colluvium sp. nov. isolated from conifer litter. Mycologia 87(4), $518-524$.

Hall TA 1999 - BioEdit: a user-friendly biological sequence alignment editor and analysis program for Windows 95/98/NT. In: Nucleic Acids Symposium Series, pp 95-98

Hyde KD, Jones EBG, Liu JK, Ariyawansa H, Boehm E, Boonmee S, Braun U, Chomnunti P, Crous PW, Dai DQ, Diederich P, Dissanayake A, Doilom M, Doveri F, Hongsanan S, Jayawardena R, Lawrey JD, Li YM, Liu YX, Lücking R, Monka J, Muggia L, Nelsen MP, Pang KL, Phookamsak R, Senanayake IC, Shearer CA, Suetrong S, Tanaka K, Thambugala KM, Wijayawardene NN, Wikee S, Wu HX, Zhang Y, Begoña AH, Alias SA, Aptroot A, Bahkali AH, Bezerra JL, Bhat DJ, Camporesi E, Chukea E, Gueidan C, Hawksworth DL, Hirayama K, Hoog SD, Kang JK, Knudsen K, Li WJ, Li XH, Liu ZY, Mapook A, Mckenzie EHC, Miller AN, Mortimer PE, Phillips AJL, Raja HA, Scheuer C, Schumm F, Taylor JE, Tian Q, Tibpromma S, Wanasinghe DN, Wang Y, Xu JC, Yacharoen S, Yan JY, Zang M 2013 Families of Dothideomycetes. Fungal Diversity 63, 1-313. http://dx.doi.org/10.1007/s13225013-0263-4

Hyde KD, McKenzie EHC, KoKo TW 2011 - Towards incorporating anamorphic fungi in a natural classification - checklist and notes for 2010. Mycosphere 2, 1-88. 
http://dx.doi.org/10.5943/mycosphere/3/2/5

Index Fungorum (2015) http://www.indexfungorum.org/names/names.asp

Jayasiri SC, Hyde KD, Ariyawansa HA, Bhat DJ, Buyck B, Cai L, Dai YC, Abd-Elsalam KA, Ertz D, Gibertoni TB, Hidayat I, Jeewon R, Jones EBG, Karunarathna SC, Kirk P, Li WJ, Liu JK, Luangsa-ard JJ, Maharachchikumbura SSN, Manamgoda DS, McKenzie EHC, Moncalvo JM, Ghobad-Nejhad M, Nilsson H , Pang KL, Pereira OL, Raspé O, Rollins AW, Romero AI, Salazar JAE, Stephenson S, Suetrong S, Taylor JE, Tsui CKM, Vizzini A, Abdel-Wahab MA, Wen TC, Boonmee S, Dai DQ, Daranagama DA, Dissanayake AJ, Ekanayaka AH, Hongsanan S, Jayawardena RS, Perera RH, Phookamsak R, De Silva NI, Thambugala KM, Tian Q, Wanasinghe DN, Wijayawardene NN, Zhao RL, Kang JC, Promputtha I 2015 - The Faces of Fungi database-A unique perspective: Fungal names linked with morphology, phylogeny and human impacts. Fungal Diversity (in press). http://dx.doi.org/10.1007/s13225015-03518-8

Kirk PM, Cannon PF, Minter DW 2010 - Dictionary of the Fungi 10th ed.-CABI (2008)2.

Li WJ, Liu JK, Bhat DJ, Camporesi E, Dai DQ, Mortimer PE, Xu JC, Hyde KD, Chomnunti P 2015 Molecular phylogenetic analysis reveals two new species of Discosia. Phytotaxa 203, 37-46. http://dx.doi.org/10.11646/phytotaxa.181.1.1

Liu JK, Hyde KD, Jones EBG, Ariyawansa HA, Bhat DJ, Boonmee Maharachchikumbura SSN, Mckenzie EHC, Phookamsak R, Phukhamsakda C, Shenoy BD, Abdel-Wahab MA, Buyck B, Chen J, Chethana KWT, Singtripop C, Dai DQ, Dai YC, Daranagama DA, Dissanayake AJ, Doliom M, D’Souza MJ, Fan XL, Goonasekara ID, Hirayama K, Hongsanan S, Jayasiri SC, Jayawardena RS, Karunarathna SC, Li WJ, Mapook A, Norphanphoun C, PANG KL, Perera RH, Peršoh D, Pinruan U, Senanayake IC, Somrithipol S, Suetrong S, Tanaka K, Thambugala KM, Tian Q, Tibpromma S, Udayanga D, Wijayawardene NN, Wanasinghe D, Wisitrassameewong K, Abdel-Aziz FA, Adamčík S, Bahkali AH, Boonyuen N, Bulgakov T, Callac P, Chomnunti p, Greiner K, Hashimoto A, Hofstetter V, Kang JC, Lewis D, Li XH, Liu XX, Liu ZY, Matumura M, Mortimer PE, Rambold G, Randrianjohany E, Sato G, SriIndrasutdhi V, Tian CM, Verbeken A, Von Brackel W, Wang Y, Wen TC, Xu JC, Yan JY, Zhao RL,Camporesi E. 2015 - Fungal diversity notes 1-110: taxonomic and phylogenetic contributions to fungal species. Fungal Diversity 72 (1), 1-197. http://dx.doi.org/10.1007/s13225-015-0324-y

Page RDM 1996 - TreeView: an application to display phylogenetic trees on personal computers. Computer Applications in the Biosciences 12, 357-358.

Phookamsak R, Liu JK, McKenzie EHC, Manamgoda DS, Ariyawansa H, Thambugala KM, Dai DQ, Camporesi E, Chukeatirote E, Wijayawardene NN, Bahkali AH, Mortimer PE, Xu JC, Hyde KD. 2014 - Revision of Phaeosphaeriaceae. Fungal Diversity 68, 159-238. http://dx.doi.org/10.1007/s13225-014-0308-3

Phookamsak R, Norphanphoun C, Tanaka K, Dai DQ, Luo ZL, Liu JK, Su HY, Bhat DJ, Bahkali Ah, Mortimer PE, Xu JC, Hyde 2015 - Towards a natural classification of Astrosphaeriella-like species; introducing Astrophaeriellaceae and Pseudoastrosphaeriellaceae fam. nov. and Astrosphaeriellopsis, gen. nov. 2015 - Fungal Diversity (in press)

Quaedvlieg W, Verkley GJM, Shin H-D, Barreto RW, Alfenas AC, Swart WJ, Groenewald JZ, Crous PW 2013 - Sizing up Septoria. Studies in Mycology 75, 307-390. http://dx.doi.org/ $10.3114 / \operatorname{sim} 0017$.

Schoch CL, Crous PW, Groenewald JZ, Boehm EWA, Burgess TI, de Gruyter J, de Hoog GS, Dixon LJ, GrubeM, Gueidan C, Harada Y, Hatakeyama S, Hirayama K, Hosoya T, Huhndorf SM, Hyde KD, Jones EBG, Kohlmeyer J, Kruys Å, Li YM, Lücking R, Lumbsch HT, Marvanová 
L, Mbatchou JS,McVay AH,MillerAN, Mugambi GK,Muggia L, Nelsen MP, Nelson P, Owensby CA, Phillips AJL, Phongpaichit S, Pointing SB, Pujade-Renaud V, Raja HA, Rivas Plata E, Robbertse B, Ruibal C, Sakayaroj J, Sano T, Selbmann L, Shearer CA, Shirouzu T, Slippers B, Suetrong S, Tanaka K, Volkmann-Kohlmeyer B, Wingfield MJ, Wood AR, Woudenberg JHC, Yonezawa H, Zhang Y, Spatafora JW 2009 - A class-wide phylogenetic assessment of Dothideomycetes. Studies in Mycology 64, 1-15. http://dx.doi.org/10.3114/sim.2009.64.01

Silvestro D, Michalak I. 2011 - raxmlGUI: a graphical front-end for RAxML. Organisms Diversity and Evolution 12: 335-337. http://dx.doi.org/10.1007/s13127-011-0056-0

Sutton BC 1980 - The Coelomycetes-Fungi imperfecti with pycnidia, acervuli and stromata. Commonwealth Mycological Institute, Kew, UK, 496 pp.

Thompson JD, Gibson TJ, Plewniak F, Jeanmougin F, Higgins DG 1997 - The CLUSTAL_X windows interface: flexible strategies for multiple sequence alignment aided by quality analysis tools. Nucleic Acids Research 25(24), 4876-4882.

Trakunyingcharoen T, Lombard L, Groenewald JZ, Cheewangkoon R, Toanun C, Alfenas AC, Pedro W Crous. 2014 - Mycoparasitic species of Sphaerellopsis, and allied lichenicolous and other genera. IMA Fungus ·5(2), 391-414. http://dx.doi.org/10.5598/imafungus.2014.05.02.05

Vilgalys R, Hester M. 1990 - Rapid genetic identification and mapping of enzymatically amplified ribosomal DNA from several Cryptococcus species. Journal of Bacteriology 172:4238-4246.

Wanasinghe DN, Jones EBG, Camporesi E, Boonmee S, Karunarathna SC, Thines M, Mortimer PE, Xu JC, Hyde KD 2014 - Dematiopleospora mariaegen. sp. nov., from Ononis spinosa in Italy. Cryptogamie, Mycologie 35 (2), 105-117. http://dx.doi.org/10.7872/crym.v35.iss2.2014.105

White TJ, Bruns T, Lee S, Taylor J 1990 - Amplification and direct sequencing of fungal ribosomal RNA genes for phylogenetics. In: Innis MA, Gelfand DH, Sninsky JJ, White TJ (eds) PCR protocols: a guide to methods and applications. Academic, San Diego, pp 315-322

Wijayawardene DNN, Crous PW, Kirk PM, Hawksworth DL, Boonmee S, Braun U, Dai DQ, D'souza MJ, Diederich P, Dissanayake A, Doilom M, Hongsanan S, Jones EBG, Johannes Z, Groenewald, Jayawardena R, Lawrey JD, Liu JK, Lücking R, Madrid H, Manamgoda DS, Muggia L, Nelsen MP, Phookamsak R, SuetrongS, Tanaka K, Thambugala KM, Wanasinghe DN, Wikee S, Zhang Y, Aptroot A, Ariyawansa HA, Bahkali AH, Bhat DJ, Gueidan C, Chomnunti P, De Hoog GS, Knudsen K, Li WJ, McKenzie EHC, Miller AN, Phillips AJL, Piątek M, Raja HA, Shivas RS, Slippers B, Taylor JE, Tian Q, Wang Y, Woudenberg JHC, Cai L, Jaklitsch WM, Hyde KD 2014 - Naming and outline of Dothideomycetes - 2014 including proposals for the protection or suppression of generic names. Fungal Diversity 69,155. http://dx.doi.org/10.1007/s13225-014-0309-2

Wijayawardene DNN, McKenzie EHC, Hyde KD. 2012b - Towards incorporating anamorphic fungi in a natural classification-checklist and notes for 2011. Mycosphere 3(2): 157-228. http://dx.doi.org/10.5943/mycosphere/3/2/5

Wijayawardene DNN, Song Y, Bhat DJ, McKenzie EHC, Chukeatirote E, Wang Y, Hyde KD. 2013 Wojnowicia viburni sp. nov., from China and its phylogenetic placement. Sydowia 65:129138.

Zhang Y, Crous PW, Schoch CL, Hyde KD. 2012 - Pleosporales. Fungal Diversity 53:1-221. http://dx.doi.org/10.1007/s13225-011-0117-X

Zhang Y, Schoch CL, Fournier J, Crous PW, de Gruyter J, Woudenberg JHC, Hirayama K, Tanaka K, Pointing SB, Spatafora JW, Hyde KD 2009 - Multi-locus phylogeny of Pleosporales: a taxonomic, ecological and evolutionary re-evaluation. Studies in Mycology 64:85-102. http://dx.doi.org/10.3114/sim.2009.64.04 